THE THERMAL ION DYNAMICS EXPERIMENT AND PLASMA SOURCE INSTRUMENT

\author{
T. E. MOORE, C. R. CHAPPELL, M. O. CHANDLER, S. A. FIELDS, \\ C. J. POLLOCK and D. L. REASONER * \\ Marshall Space Flight Center, Huntsville, AL 35812, U.S.A. \\ D. T. YOUNG, J. L. BURCH, N. EAKER and J. H. WAITE, JR. \\ Southwest Research Institute, San Antonio, TX 78284, U.S.A. \\ D. J. McCOMAS, J. E. NORDHOLDT and M. F. THOMSEN \\ Los Alamos National Laboratory, Los Alamos, NM 87545, U.S.A. \\ J. J. BERTHELIER \\ Centre de Recherches en Physique de l'Environment, St. Maur des Fosses, France 94107 \\ and \\ R. ROBSON \\ Hughes Aircraft Co., Research Laboratories, Malibu, CA 90265, U.S.A.
}

(Received 30 March, 1993)

\begin{abstract}
The Thermal Ion Dynamics Experiment (TIDE) and the Plasma Source Instrument (PSI) have been developed in response to the requirements of the ISTP Program for three-dimensional (3D) plasma composition measurements capable of tracking the circulation of low-energy $(0-500 \mathrm{eV})$ plasma through the polar magnetosphere. This plasma is composed of penetrating magnetosheath and escaping ionospheric components. It is in part lost to the downstream solar wind and in part recirculated within the magnetosphere, participating in the formation of the diamagnetic hot plasma sheet and ring current plasma populations. Significant obstacles which have previously made this task impossible include the low density and energy of the outflowing jonospheric plasma plume and the positive spacecraft floating potentials which exclude the lowest-energy plasma from detection on ordinary spacecraft. Based on a unique combination of focusing electrostatic ion optics and time of flight detection and mass analysis, TIDE provides the sensitivity (seven apertures of $\sim 1 \mathrm{~cm}^{2}$ effective area each) and angular resolution $\left(6^{\circ} \times 18^{\circ}\right)$ required for this purpose. PSI produces a low energy plasma locally at the POLAR spacecraft that provides the ion current required to balance the photoelectron current, along with a low temperature electron population, regulating the spacecraft potential slightly positive relative to the space plasma. TIDE/PSI will: (a) measure the density and flow fields of the solar and terrestrial plasmas within the high polar cap and magnetospheric lobes; (b) quantify the extent to which ionospheric and solar ions are recirculated within the distant magnetotail neutral sheet or lost to the distant tail and solar wind; (c) investigate the mass-dependent degree energization of these plasmas by measuring their thermodynamic properties; (d) investigate the relative roles of ionosphere and solar wind as sources of plasma to the plasma sheet and ring current:
\end{abstract}

\title{
Introduction
}

The Thermal Ion Dynamics Experiment/Plasma Source Instrument derives from a broad range of experience with low-energy plasma measurements at high altitude extending from some of the earliest measurements of the plasmasphere on

* Deceased. 
OGO-5, including the ATS-6, GEOS, SCATHA, and ISEE plasma instruments, and culminating most recently with the Retarding Ion Mass Spectrometer (RIMS) on Dynamics Explorer 1 (DE 1), which operated between September 1981 and February 1991 (Chappell et al., 1981; Chappell, 1988). These Instruments measured angular and energy characteristics by mass species, revealing that non-thermal features often characterize the 'thermal plasma', and leading to a new appreciation of the three-dimensionality of plasma flows, including field-aligned bulk motions of the ionosphere.

Two fundamental lessons were learned from these earlier experiences: (1) highaltitude spacecraft carry plasma instruments into regions of very low plasma density where the range of geometric factors (combined area and solid angle response) previously considered adequate for plasma instrumentation (e.g., $\sim 10^{-2} \mathrm{~cm}^{2}$, $1 \mathrm{sr}$ ), becomes inadequate; (2) positive floating potentials of spacecraft exposed to sunlight and low-density plasmas make low-energy plasma observations all but impossible by excluding low-energy ions from the spacecraft, exacerbating the sensitivity problem. Experience with the ATS-6 and SCATHA spacecraft (Olsen, 1981,1985 ) has shown that spacecraft floating potential can be regulated at small values by means of the operation of a low-energy plasma source on the spacecraft. It should be noted that both of these problems are peculiar to low-energy plasma measurements, because the flux of hot plasma particles is relatively unaffected by floating potential effects and is larger than for a similar density of low-energy particles. Consequently, hot plasma ions are more readily observable than an equal density of very low-energy ions.

\section{Science Objectives}

The Thermal Ion Dynamics Experiment (TIDE) and Plasma Source Instrument (PSI) have been developed in response to the requirements of the ISTP program for three-dimensional (3D) plasma composition measurements capable of tracking the circulation of low-energy plasmas throughout the polar magnetosphere. Large and relatively steady outflows of low energy ionospheric heavy ions $\mathrm{CO}^{++}, \mathrm{O}^{+}$, $\mathrm{N}^{+}$, and, to a lesser extent, $\mathrm{N}_{2}^{+}, \mathrm{NO}^{+}$, and $\mathrm{O}_{2}^{+}$) were identified by the Dynamics Explorer 1 Retarding Ion Mass Spectrometer (DE 1/RIMS) as originating in the auroral zone region (Waite et al., 1985; Moore et al., 1985; Lockwood et al., 1985; Pollock $e t a l ., 199(1)$, particularly from the dayside. Relatively steady outflows of light ionospheric ions $\left(\mathrm{H}^{+}, \mathrm{He}^{+}\right)$were identified as originating from the entire high latitude region (Nagai et al., 1985; Chandler et al., 1991). This plasma is in part lost into the downstream solar wind and in part recirculated within the inner magnetosphere, participating in the formation of the diamagnetic hot plasma sheet and ring current plasma populations (Moore ot al., 1990; Delcourt et al., 1990), as well as the outer plasmasphere. 
More recently (Giles et al., 1994) it has been discovered that the auroral zone is a significant source of $m / Z=2$ ions. Whereas such ions occur at an abundance relative to $\mathrm{H}^{+}$of order $10^{-3}$ in the cold ionosphere (consistent with cosmic Deuterium abundance), the relative flux of such ions in auroral beams and conics is up to two orders of magnitude higher. This strongly suggests that $\mathrm{He}^{++}$is being created by the energetic electron precipitation of the auroral zone, calling into question the validity of source contribution inferences based on $\mathrm{He}^{++}$composition. The fact that the ionosphere is a significant source of the major solar wind species introduces an ambiguity that can only be resolved by establishing unambiguously the transport of the ionospheric and solar plasmas within the magnetosphere.

The nominal $2 \times 9 R_{\mathrm{E}}$ orbit of the POLAR spacecraft will project it high over the polar caps in the lobes of the magnetotail where it will be perfectly positioned to sample the continuation of the ionospheric outflows into the nightside magnetosphere as well as the entrainment of solar wind plasma into magnetospheric circulation. However, as noted above, the high altitude and low plasma densities present an observational challenge.

TIDE provides the sensitivity (seven channels having effective apertures approaching $1 \mathrm{~cm}^{2}$ each) and angular resolution required for this purpose. PSI produces a low-energy plasma locally at the POLAR spacecraft, providing the ion current required to balance the photoelectron current, along with a low temperature electron population, thus providing for the regulation of the spacecraft potential slightly positive relative to the space plasma.

Thus TIDE/PSI will address the following objectives:

(a) To measure the position-dependent density and flow velocity field of the polar wind, the heated auroral zone plasma outflows, and the low energy component of magnetosheath plasmas $(<500 \mathrm{eV})$ in the high polar cap and magnetospheric lobes.

(b) To quantify the extent to which ionospheric and solar ions are recirculated within the distant magnetotail neutral sheet or lost to the distant tail and solar wind.

(c) To investigate the mass dependent degree of energization achieved as plasma flows anti-sunward on merged flux tubes through the polar cap and into the lobes, by measuring the thermodynamic properties of these plasmas.

(d) To investigate the relative roles of ionosphere and solar wind as sources of plasma to the plasma sheet and ring current, by identifying the characteristic populations and distinguishing their behaviors using measurements of composition, flow, and thermodynamic properties.

\section{TIDE SPECIFICATIONS}

The fundamental design goal for TIDE has been to achieve a geometric factor much greater than that of previous instruments, opening up an unknown regime to discovery. An extremely large dynamic range of low-energy plasma fluxes is seen 
by a spacecraft in the course of an orbit from the ionosphere to the polar regions at several RE altitude. DE 1/RIMS, for example, routinely experienced fluxes which ranged from well above detector saturation levels to low levels requiring long accumulations, to levels so low they could not be measured above an extremely low detector noise rate $(\leq 1 \mathrm{~Hz})$. Therefore, though POLAR will not travel as close to the Earth as DE 1 did, extension of dynamic range is required in order to adequately sample both the lowest and highest density/flux regions.

TIDE has also been designed to provide low-energy measurements which are differential in energy and direction as well as mass, with range and resolution adequate for the full characterization of velocity distribution features known to exist in the low-energy plasma populations. These features include polar wind flows of very high Mach number, which were unresolved in angle or energy by DE 1/RIMS. Other features, that appeared to DE 1 as crosswinds, could be lost between the RIMS fields of view due to insufficient angular coverage. Another important type of feature is the angle-dependent energy distribution of transversely accelerated ion distributions or ion conics, which required differentiation of the RIMS Retarding Potential Analyzer (RPA) curves with a resulting loss of signal to noise ratio.

Time resolution is of primary importance on a rapidly moving spacecraft when it passes through localized structures. The larger the number of variables scanned during instrument operations, the more difficult it becomes to achieve adequate temporal resolution of phenomena. This problem is all the more serious for mass spectrometers. Prototypes of TIDE used an electrostatic deflection system for angular scanning with a single mass analyzer section. It has become apparent during sounding rocket flights of such instruments (e.g., the SuperThermal Ion Composition Spectrometer or STICS) that this provides an imperfect solution to the problem of angular range, placing extreme requirements on the stepping power supplies which sweep the angle, energy, and mass selection. TIDE solves this problem by using multiple independent angular detection channels, each equipped with an independent time-of-flight (TOF) mass analyzer section, which monitors all mass species simultaneously. The only variable which must be swept, using stepping power supplies, is the ion energy. This can be accomplished in a time small compared with the POLAR spin period, so that single spin time resolution of the multi-species 3D distribution function is achieved.

Table I provides a summary of the technical specifications for TIDE which details the resolution and sensitivity of the instrument. TIDE's geometric factor is nearly an order of magnitude larger than that of DE/RIMS on a 'per channel' basis. Moreover, TIDE has seven simultaneously-active contiguous channels, compared with three separated channels for RIMS. It can be argued that a more meaningful figure of merit for the sensitivity of an instrument than the geometric factor is the 'effective area' of the aperture. This measure does not award credit for large solid angle or energy apertures, which degrade the resolution of the instrument. By this measure, each TIDE aperture is nearly a full two orders of magnitude larger than a 
TABLE I

TIDE specifications

\begin{tabular}{ll}
\hline Parameter & Value \\
\hline Energy range & $0.1-500 \mathrm{cV}$ \\
Energy resolution & $5 \%-100 \%$, commandable \\
Mass range & $1-40 \mathrm{amu}$ \\
Mass resolution & $M / \Delta M=4$ at FWHM \\
Angular coverage & sampling: $11.25^{\circ} \times 22.5^{\circ} \times 7$ sectors \\
& per spin: $360^{\circ} \times 157.5^{\circ}$ in $32 \times 7$ samples \\
Angular resolution/response & $6^{\circ} \times 18^{\circ}=0.033$ steradians \\
Total field of view & $96 \%$ of $4 \pi$ sr \\
Nominal pixels per image & $35 \mathrm{k}=32$ energy $\times 32$ Az $\times 7$ polar $\times 5$ mass \\
Aperture (non-mass-analyzed) & $\lesssim 1 \mathrm{~cm}^{2}$ per sector $\times 7$ sectors \\
Geometric factor (non-mass-analyzed) & $\lesssim 0.03 \mathrm{~cm} \mathrm{~s}^{2}$ sr per sector $\times 7$ sectors \\
Time resolution 1 sample & $5.86 \mathrm{~ms}$ \\
Time resolution 2D & $\frac{1}{32} \mathrm{spin}$ pcriod $(0.1875 \mathrm{~s})$ \\
Time resolution 3D & $1 \mathrm{spin}$ period $(6 \mathrm{~s})$ \\
Dynamic range & $10^{8}$ using variable $\Delta E / E$ and aperture \\
& \\
Resources & $17.1 \mathrm{~kg}$ \\
Mass & $9.1 \mathrm{~W}$ \\
Power & $4.0 \mathrm{kbps}$ \\
Telemetry &
\end{tabular}

RIMS aperture (and outnumber them $7: 3$ ), having an effective area approaching $1 \mathrm{~cm}^{2}$. Such a large effective area allows TIDE to be designed for improved energy and angular resolution, while maintaining very high sensitivity.

\section{PSI SPECIFICATIONS}

The fundamental goal of PSI is to establish a means for the reduction of the spacecraft floating potential to values much smaller than normally associated with spacecraft in low-density plasmas in sunlight. The Plasma Source Instrument (PSI) is an essential adjunct to TIDE, because control of the potential of the POLAR spacecraft will allow measurements of low- energy ions in regions of very low plasma density. Spacecraft immersed in plasmas in general attain a potential different from the plasma potential.

The origins of this spacecraft potential are complex, but the fundamental principle is that the net current to the spacecraft surface has to be zero in steady state. The currents flowing between the spacecraft and the plasma are all functions of this potential, and the potential adjusts self-consistently, on very short time scales, 
to changes in plasma environment or solar irradiance so as to satisfy a zero-current condition. Equilibrium spacecraft potentials can range from several volts positive (to retain emitted photoelectrons) when the spacecraft is in a low-density plasma, to thousands of volts negative when the spacecraft is simultaneously in the shadow of Earth and in a very hot plasma such as is found in the plasma sheet during substorm activity.

A charged particle starting far from the spacecraft and arriving at the spacecraft surface is deflected from its initial direction and arrives at an energy which differs from its initial energy owing to spacecraft floating potential. lons approaching a positively charged spacecraft with insufficient energy are prevented from reaching the instrument, leading to an excluded sphere in velocity space and centered on the spacecraft frame, within which the velocity distribution cannot be measured. Negative floating potentials raise the apparent energy of the ions and may preclude the implementation an energy sampling strategy which is effective in resolving the ambient energy distribution.

Experience with plasma source and accelerator devices on the ATS-6 and SCATHA spacecraft has shown that it is feasible to maintain the spacecraft floating potential at small values in the presence of changes in external plasma or sunlight conditions. This is done by establishing a plasma in the vicinity of the spacecraft with the following properties:

(a) The generated plasma must have an ion thermal flux which is larger than the electron thermal flux of the photoelectron cloud generated by the incident EUV flux on the spacecraft surfaces. In addition, the local plasma electron thermal flux must be larger than that of any naturally occurring plasma expected to be encountered by the spacecraft.

(b) The generated plasma electron temperature is small, a few tenths of eV at most.

(c) The generated plasma ions are chemically inert, with a mass per charge which is distinct from that of naturally occurring ions.

Condition (a) insures that the thermal flux of local plasma ions is capable of neutralizing the flux of photoelectrons away from the spacecraft which results if the spacecraft floating potential is maintained at values much less than the mean energy of the photoelectrons. Condition (a) further insures that the thermal flux of local plasma electrons is sufficient to neutralize the flux to the spacecraft of very hot electrons frequently encountered near synchronous orbit. Condition (b) insures that the equilibrium floating potential is much smaller than either the several volts corresponding to the photoelectron mean energy, or the thousands of volts corresponding to plasma sheet electron temperatures. Condition (c) insures that the operation of the plasma source does not create a plasma which can be confused with the ambient plasma.

The PSI design of a source which produces a suitable plasma is based on earlier experiences with ATS-6 and SCATHA. This design has been developed by the Hughes Aircraft Corp. under a contract with the U.S. Air Force. Flight hardware 
TABLE II

PSI specifications

\begin{tabular}{ll}
\hline Parameter & Value \\
\hline $\begin{array}{l}\text { Plasma ion thermal flux } \\
\text { Plasma electron thermal flux }\end{array}$ & $\begin{array}{l}\text { exceeds } 25 \mu \mathrm{A} \text { (actual capacity: } 1 \mathrm{~mA} \text { ) } \\
\text { exceeds that of plasma: } 3 \mathrm{~cm}^{-3} \text { with } T=5 \mathrm{keV} \\
\text { Plasma ion species }\end{array}$ \\
$\begin{array}{l}\text { Xenon } \\
\text { Resources: }\end{array}$ & $20000 \mathrm{hr}$ \\
Operational life & $15 \mathrm{~W}$ \\
Operating power & $45 \mathrm{~W}$ \\
Startup power (5 min each start) &
\end{tabular}

has been previously produced, tested, and flown by Hughes in connection with these programs, and the design is mature. Table II provides a summary of the technical specifications for the PSI which details the capabilities of the system.

\section{TIDE Instrument Description}

Plate 1 shows a photograph of the TIDE sensor apertures and TOF/detector system, with the cylindrical sensor cover removed. Figure 1 shows an exterior assembly view of the TIDE sensor with all covers in place. The sensor and electronics are integrated into a single unit. Routine access to the sensor components requires removing only the outer cylindrical cover. Routine access to the electronics boards requires removing only the rear panel on the electronics compartment. The outer surfaces of the instrument that are in contact with the plasma are plated with electroless nickel. Optical surfaces within the sensor are treated, for reduced EUV reflectance, with cupric oxide black (Ebanol-C). Other surfaces are polished or bead-blasted aluminum, where scattering of light and particles is not a problem.

The sensor is designed with a housing which seals tightly to serve as a diffusion barrier against chemical, water vapor, and dust contamination of the sensor interior. TIDE's detectors are sensitive to hydrocarbon contamination and are also strongly hygroscopic. The sealed sensor housing is kept backfilled with gaseous high purity nitrogen. The apertures and other vent openings are fitted with purge covers which are removed before flight. The apertures are covered until orbital operations by a conductively treated Kapton sheet which is retracted onto a double negator springactuated spindle at the appropriate time by remote command.

Figure 2 shows the relative location and orientation of the TIDE sensor and the PSI source on the spacecraft, with respective fields of view indicated. The TIDE field of view is a segmented fan coplanar with the spacecraft spin axis and one 


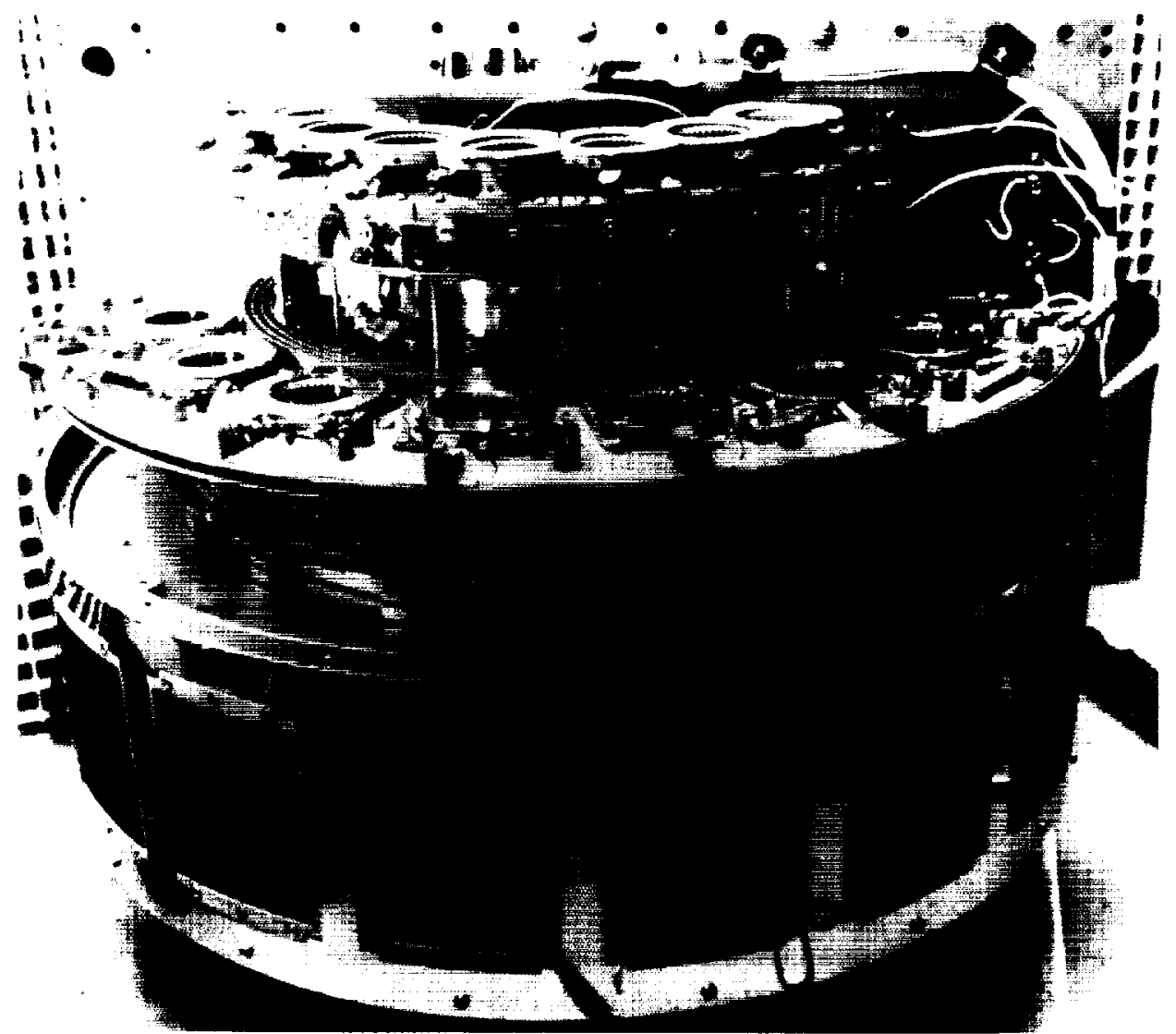

Plate 1. Photograph of TIDE sensor illustrating the orientation of the seven entrance apertures with respect to the semi-cylindrical instrument package, and the layout of assemblies on the sensor baseplate.

edge of the field of view is aligned with the spin axis. TIDE's seven polar angle sectors are arranged in a fan spanning $157.5^{\circ}$, as shown in Figures 2 and 3. Each angular sector contains a collimator, electrostatic mirror, and RPA, as shown in Figure 4. The electrostatic mirrors have an off-axis corrected parabolic form and are preceded by collimators that eliminate extraneous particles and photons at the aperture of each sector. Each electrostatic mirror sets an upper energy limit, since ions are only reflected and focused if their energy is below a cutoff set by the mirror potential (and to a lesser extent their incidence angle). Following the mirror is a RPA, similar in operating principle to that of DE-1/RIMS, which independently sets a lower limit on the ion energy. The net result is an electronically-controlled differential energy pass band.

Coupled with each mirror/RPA (M/RPA) system is an EUV-rejection/deflection system and TOF mass analyzer system with electronics similar to that developed for the LOMICS instrument on the CRRES mission (Young, 1989). After coming to a focus at the entrance to the rejection/deflection section (at chassis ground), ions 


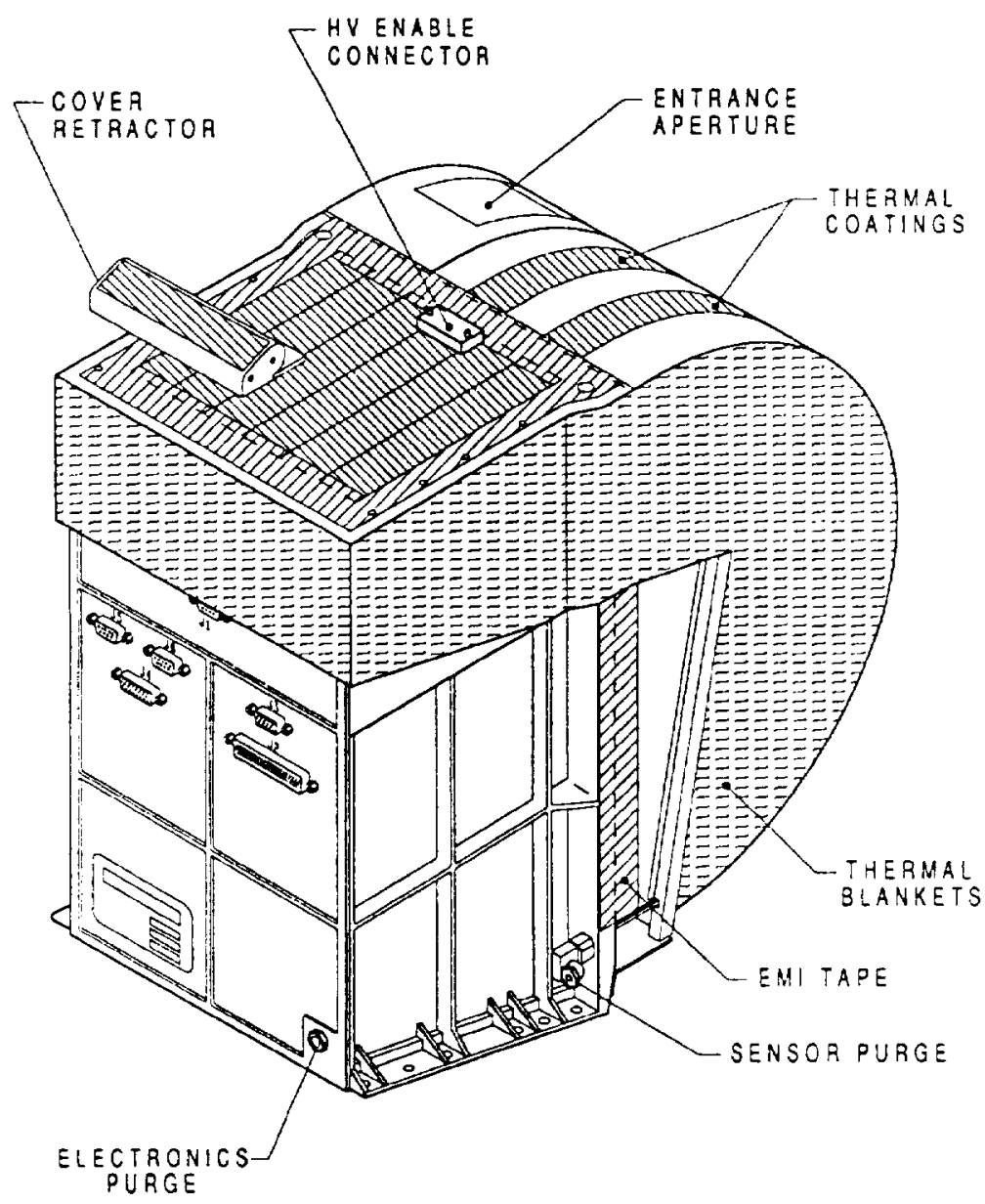

Fig. 1. Perspective view of TIDE instrument including its electronics package, showing the orientation of entrance apertures, arming plugs, thermal blankets, purgefittings, connectors, and mounting lugs.

are first accelerated as they pass through a second aperture at $-3.0 \mathrm{kV}$, then further accelerated to and simultaneously deflected radially inward within the sensor by a UV-rejection/deflection system. The ions are then deflected back outward and accelerated to $-12 \mathrm{kV}$ so as to enter the TOF start gate traveling parallel to the direction in which they entered the rejection/deflection system. The ions enter the TOF start gate by passing through thin $\left(<1 \mu \mathrm{g} \mathrm{cm}^{-2}\right)$ carbon foils. Secondary electrons produced on the foil exit surface are collected at the START microchannel plates (MCPs) to form a 'start' pulse for the TOF electronics. The ions then traverse the TOF region and arrive at the STOP MCPs where a 'stop' pulse is generated for the TOF electronics. The delay between start and stop signals is then converted into a voltage which is inversely proportional to velocity at known 


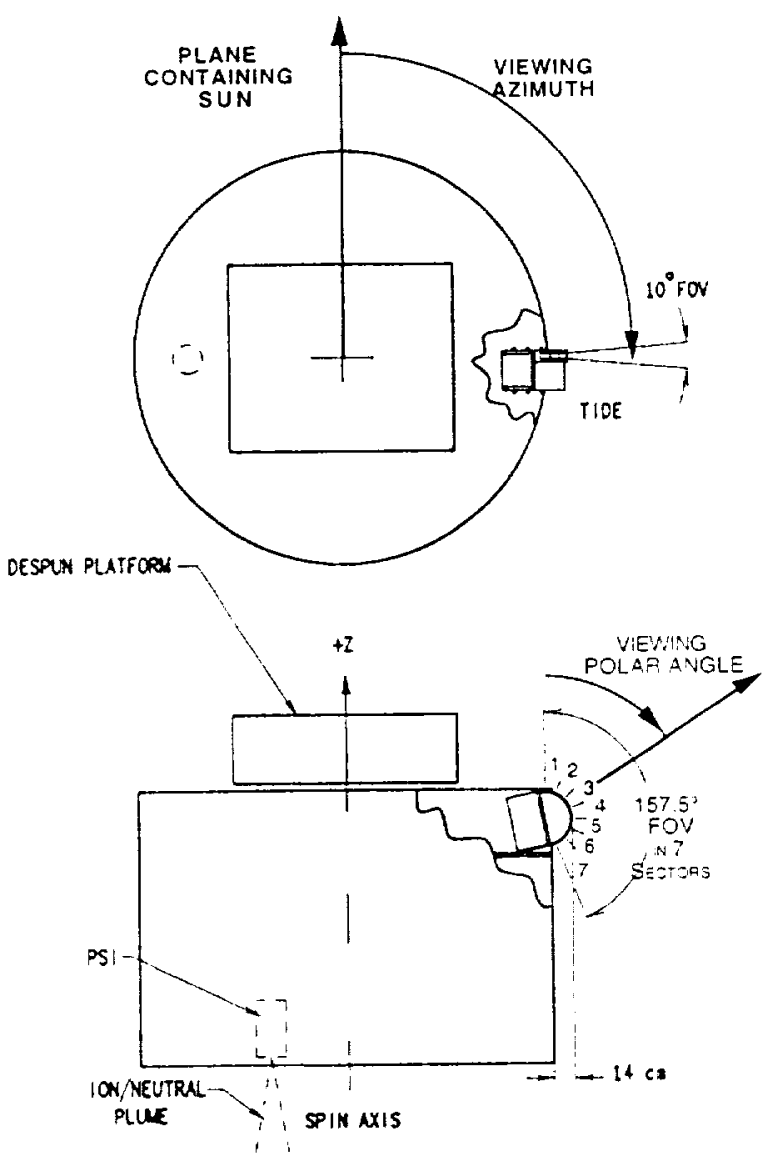

Fig. 2. Two views of the POLAR spacecraft showing TIDE and PSI pointing and mounting layout. Note that one TIDE aperture field of view is contiguous with the spin axis.

energy/charge, and therefore can be converted to a digital mass/charge address word (8-bit resolution).

When used in conjunction with a sufficient pre-acceleration potential $(-12 \mathrm{kV})$, the TOF technique has no intrinsic low-energy limit for mass analysis, leading to a straightforward application of TOF to the TIDE investigation. Unlike magnetic mass analysis systems, the TOF technique permits the use of large entrance apertures, since no object slit is required to form a mass spectrum image. The large pre-acceleration potential also allows the use of a very large angular acceptance aperture, so that very large geometric factors become possible using this technique. Similar to the operation of optical systems, the M/RPA optics transforms the TOF analyzer area and solid angle aperture to a still larger area, but smaller angular aperture, which is then presented to the external plasma. The relatively low mass of TOF devices then allows simultaneous measurement in multiple directions of all 


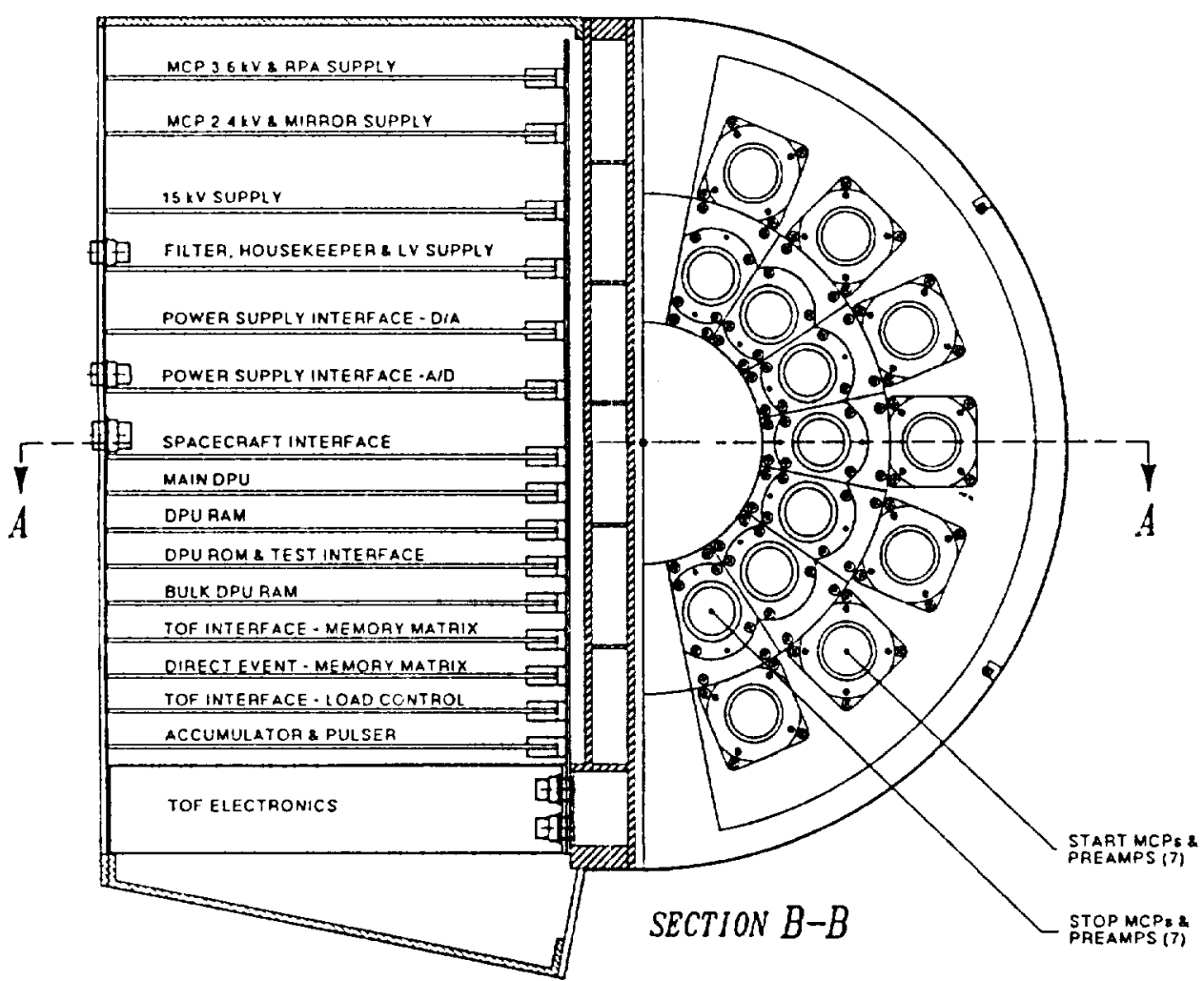

Fig. 3. Section view of the TIDE sensor with cutaways at various levels showing the placement of the START detectors and STOP detectors, as well as the angular layout of the seven channels.

ionospheric and solar species likely to be encountered by the POLAR spacecraft (viz., $\mathrm{H}^{+}, \mathrm{He}^{+}, \mathrm{He}^{++}, \mathrm{O}^{++}, \mathrm{O}^{+}$, and molecular species such as $\mathrm{NO}^{+}$).

TIDE generates very high data rates $\left(\sim 10^{5}\right.$ Bytes per satellite spin period) because of multiple look directions and simultaneous detection of all mass species. Consequently, a powerful onboard data processing unit (DPU) is required. This DPU is based on a pair of SA3300 microprocessors, one to perform onboard computations and data compression, the other to control the instrument and encode the data for telemetering. Further details on each subsystem are given below. The overall functional structure of TIDE is shown in Figure 5. It should be noted that both command and data interfaces to the PSI are managed by the TIDE DPU, so that the PSI has no independent spacecraft interface.

\section{MIRROR/RPA SYSTEM}

The TIDE electrostatic mirror/RPA system provides differential energy analysis by a two-stage ion selection process. Ions which are too energetic pass through the mirror and subsequently are lost, while those of insufficient energy are reflected 
TIDE Ion Optics Raytracing

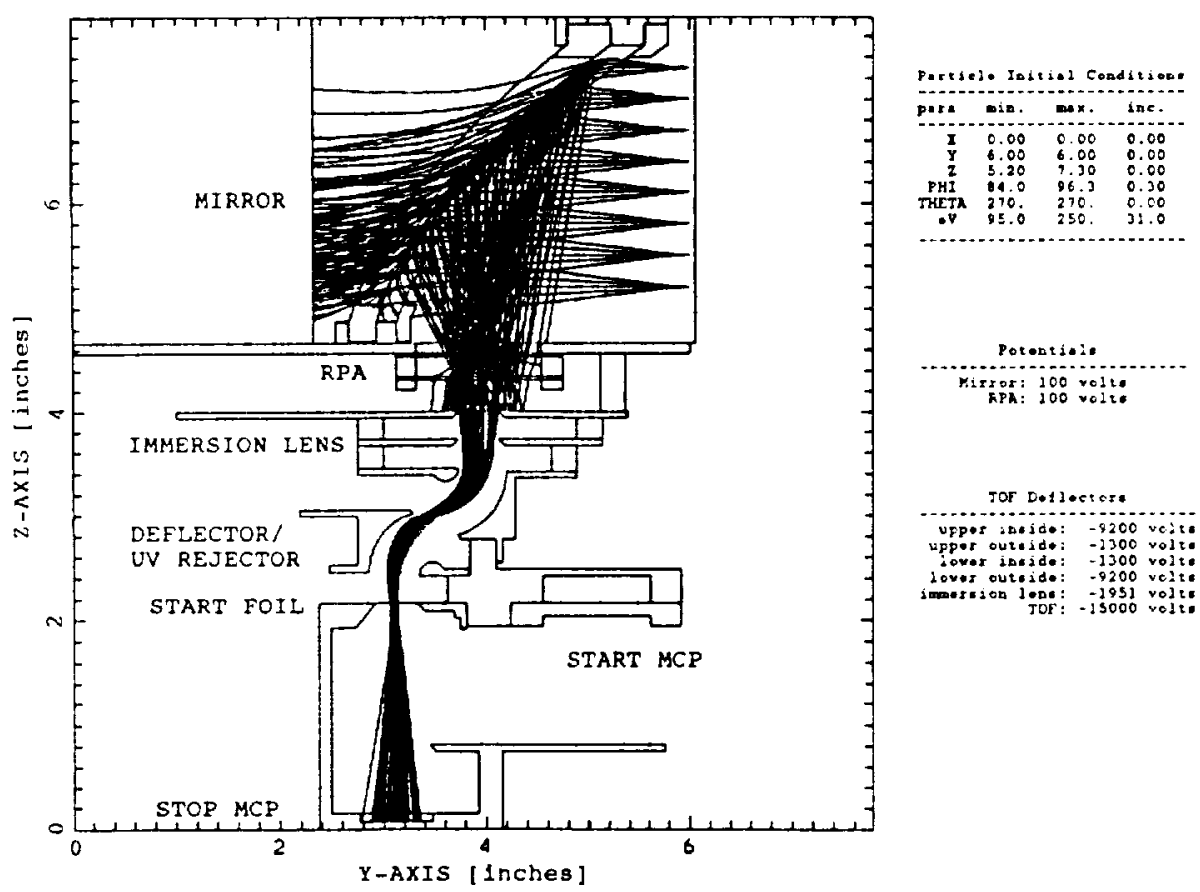

Fig. 4. Section view of the TIDE sensor showing the optics path through the mirror, RPA, immersion lens, W rejection deflector, START foils, and STOP detectors for a single channel, with rays plotted for parameters which completelyfill the instrument aperture in space, angle, and energy.

by the RPA and excluded from admittance to the TOF analyzer. Thus, ideally, a boxcar-shaped energy pass band is provided which can be adjusted in width and center energy by selection of M/RPA potentials. The actual energy response is asymmetric with a sharp cutoff on the low-energy side (due to the RPA), and a more gradual cutoff on the high-energy end (due to the mirror). Adjustment of the energy response width is used to control overall instrument sensitivity, as described below.

The mirror plays an important role in the achievement of the large TIDE geometric factor, through its focusing geometry, which is similar to the action of focusing lenses and mirrors in optical telescopes. An electrostatic mirror for charged particles consists of a biased surface suspended behind a grounded grid, and the penetration depth is in general non-negligible relative to mirror dimensions. Focusing is accomplished through the implementation of a mirror geometry which differs slightly from parabolic in a functionally-significant way. The shape is derived from the requirement that incoming ions following parallel trajectories are directed through a common focal line. This focusing action translates a large collection area 


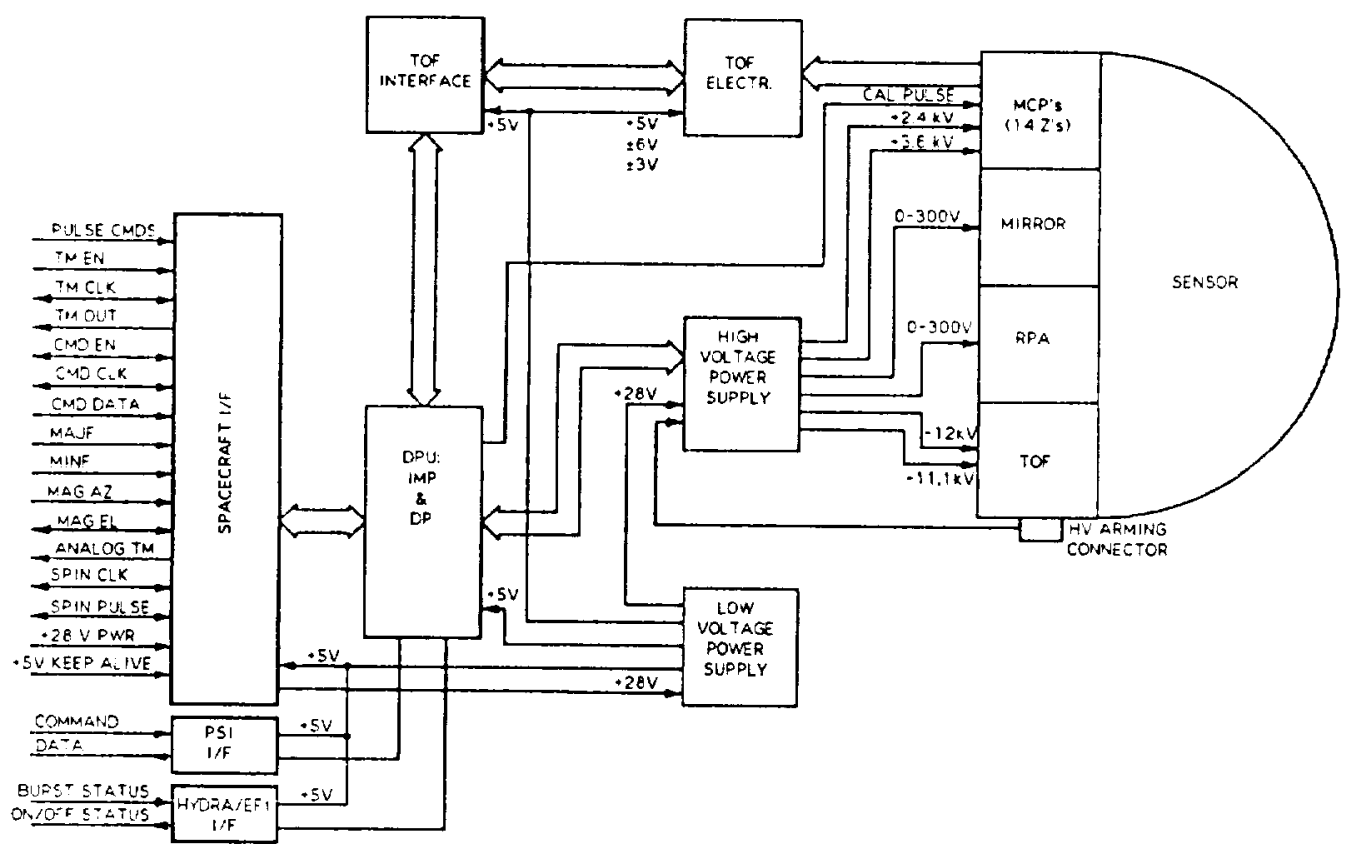

Fig. 5. TIDE functional block diagram, showing the relationships among the principal components.

and small solid angle at the entrance aperture, to a smaller collection area/larger solid angle at the entrance to the TOF section. The large solid angle acceptance at the TOF entrance is attributable to the large attractive potential encountered by ions upon entry to that section.

The RPA is a planar device consisting of four grids, as illustrated in Figure 4. The entrance and exit grids are connected to spacecraft chassis ground. The two retarding grids are mounted on opposite sides of a single conducting plate, in close proximity to entrance and exit grids, respectively. Together, they are biased at the commandable voltage $V_{\mathrm{RPA}}$. No electron suppression is included or required in view of the large negative potential separating the TIDE detection system from the RPA.

The energy resolution of the RPA system is ultimately limited by the angular spread of the ions delivered to it by the mirror system. This spread is inversely related to the breadth of the energy passband defined by the mirror and RPA, which reduces the fraction of the mirror that is active as well as the range of energies that can pass through the system.

The electrical biasing scheme employed in this system is as follows: $V_{\mathrm{RPA}}$ is commanded with a 12-bit word through a digital-to-analog converter (DAC) providing a range of $0-300 \mathrm{~V}$ with $0.073 \mathrm{~V}$ resolution. $V_{m}$ is commanded through an 8-bit DAC which is referenced to the output of the RPA DAC. In this way, the ratio $\left(V_{m} / V_{\mathrm{RPA}}\right)$ is directly commanded and can be more precisely controlled at 
the low end of the energy range than with independent commanding for both $V_{m}$ and $V_{R P A}$. This directly controls TIDE sensitivity, because the energy pass band (as well as the effective area and solid angle) is directly related to this ratio. The practical range over which TIDE's sensitivity can be varied is approximately three orders of magnitude, as described in the section below on test results.

\section{COLLIMATOR SYSTEM}

Identical collimator assemblies are installed on each of the seven TIDE polar angle apertures. These collimators consist of two stacks of vanes, one collimating in spacecraft azimuth angle, the other collimating in spacecraft polar angle. The vane stacks are nested with the polar angle stack in the outer position and the azimuth stack in the inner position. The vanes are positioned so as to eliminate all trajectories which would in any case be unacceptable to the mirror/RPA/TOF optics system; that is, all trajectories which lie outside the angular response and effective area of the instrument. This collimator approach is intended to minimize the number of extraneous particles and photons entering the instrument apertures, and particularly to eliminate such particles or photons which would otherwise be incident upon interior surfaces other than the mirror grids. In this way, the susceptibility of the instrument to extraneous particles and photons is minimized.

Collimation of $10^{\circ}$ width in spacecraft azimuth is provided by means of parallel vanes which are 0.50 in deep, 0.005 in thick, and set on 0.044 in centers. It should be noted that the M/RPA is designed for a commensurate angular response when its aperture is fully open. When the M/RPA is effectively 'stopped down' by choosing a small ratio $V_{m} / V_{\mathrm{RPA}}$ its angular response is reduced from $10^{\circ}$ to as low as $\sim 3^{\circ}$.

Collimation of $22.5^{\circ}$ in spacecraft polar angle is provided by means of vanes which are set nearly coincident with planes which emanate radially from the instrument symmetry axis. The layout of a single polar angle collimator stack is illustrated in Figure 6. Note that the individual collimator channels each fully illuminate the focal plane (rejection/deflection system entrance aperture), while cutting off rays which would be incident upon the mirror frames or other interior structures. In the figure, 'instrument $C / L$ ' refers to the symmetry axis of the sensor, around which the individual channels are arranged.

\section{REJECTION/DEFLECTION SYSTEM}

EUV-induced electron contamination of the TOF system is a very serious concern for TIDE, for in contrast to the situation with parallel curved plate electrostatic analyzers, TIDE's relatively open M/RPA system provides little rejection of EUV photons which are incident upon the mirror grid wires. The grids have a reflectivity for such photons which is the complement of the grid transmission, approximately $10 \%$ for each of the three grids, multiplied by the reflectivity of the surfaces. This 


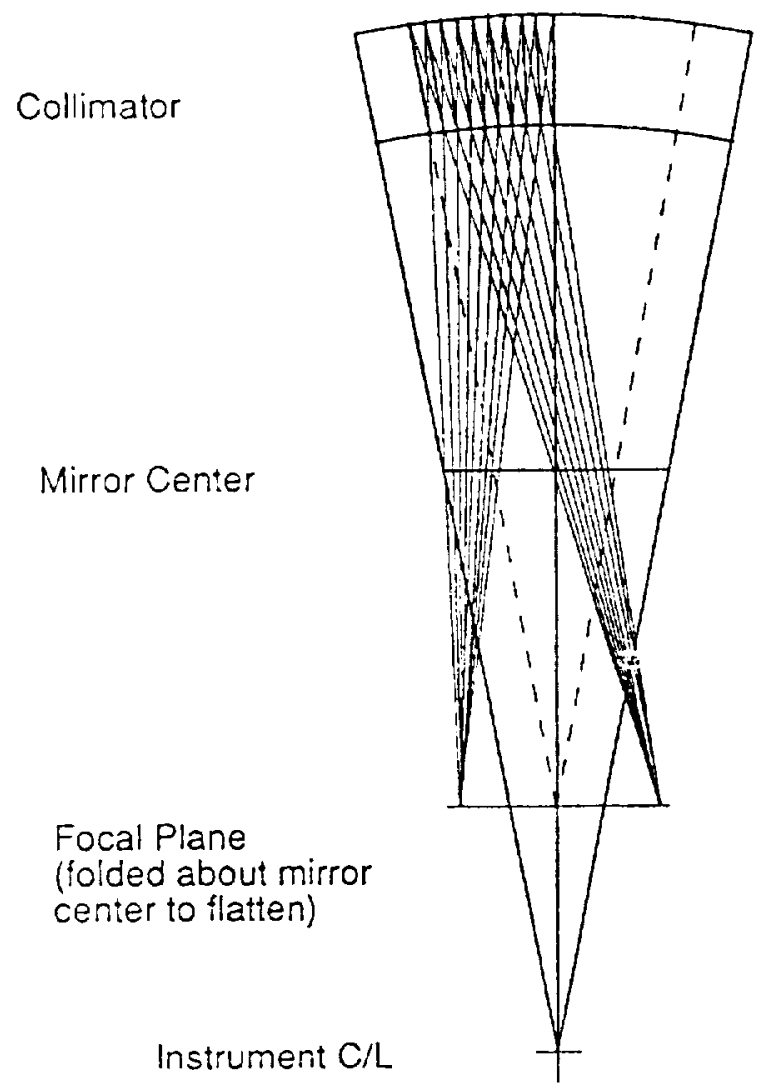

Fig. 6. Geometry for a single TIDE collimator providing $22^{\circ}$ wide response in polar angle. 'Instrument $C / L$ ' refers to the symmetry axis of the TIDE sensor, around which the polar angle channels are arranged radially, distributed in polar angle.

reflectivity is diffuse rather than specular due to the curvature and roughness of the electroformed surface. Nevertheless, measures have been taken in TIDE to introduce EUV rejection requiring at least two additional bounces between the grids and the TOF START foils. This is the purpose of the ' $\mathrm{S}$ ' shaped trajectories of ions passing from the M/RPA through the rejection/deflection system to the TOF section, as may be seen in the raytracing of Figure 4.

Displacement of the ion rays sufficient to cut off all direct paths from the mirror grids to the START foils is accomplished by means of electrodes biased so as to accelerate the ions and accomplish the two opposite deflections. The net translation is radially inward with respect to the symmetry axis of the instrument. The detailed configuration of this Rejection/Deflection section was developed by means of iterative potential solution and raytracing with the goal of accomplishing a deflection and straightening of the ion rays with as little dependence on starting 
energy as possible, over the range $0-300 \mathrm{eV}$. The result is effective in rejecting EUV photons and in preserving the bundle of rays.

\section{TOF DETECTION SYSTEM}

Ions passed by the mirror/RPA, and accelerated by the $-12 \mathrm{kV}$ potential as they pass through the Rejection/Deflection system, are incident upon an extremely thin $\left(\leq 1.0 \mu \mathrm{g} \mathrm{cm}^{-2}\right.$ ) carbon foil suspended on an electroformed grid over an aperture measuring $10 \times 25 \mathrm{~mm}$. Each ion incident upon the foil creates one or more secondary electrons of a few $\mathrm{eV}$ energy, emitted from the rear of the foil as the ion passes through. In the foil, some of the ions are charge exchanged to neutrals and suffer some degree of energy loss and angular scattering, but continue generally along their trajectories toward the first stage of the STOP MCP detectors, arriving with a time delay appropriate to their mass/charge. Electrons produced by the first stage of the STOP MCPs are accelerated by the $-12 \mathrm{kV}$ potential onto the remainder of the MCP stack where a TOF stop pulse is generated.

The electrons produced at the START foil are accelerated by the potential distribution inside the TOF analyzer. As a result, they travel sideways out of the ion flight path and are collected at the START MCP detector, which is biased at a potential more positive than that of the START foil, after a delay that is negligible in comparison with the ion time of flight from START foil to STOP foil. Other surface potentials in the vicinity of the START foils are designed so as to insure that a large fraction of the electrons emitted by the foil are detected. Sample computed electron trajectories are illustrated in Figure 7.

There are seven TOF detector systems running in parallel, one for each of the polar angle apertures. The individual START and STOP MCPs are standard circular units of $25 \mathrm{~mm}$ o.d. and $18 \mathrm{~mm}$ active diameter. The MCPs have bias angles of $\geq 5^{\circ}$ and are arranged in ' $\mathrm{Z}$ ' stacks of three individual stages per detector with pore orientations maximally out of alignment. This configuration reliably provides gain of $\geq 10^{7}$ throughout the nominal 3-yr life of the POLAR spacecraft. MCP bias supplies are commandable over a range with four bits of resolution, so that the bias can be boosted in flight to maintain sufficient gain as required. Since individual MCP stacks all share the common bias, the flight stacks will be gain matched prior to installation.

The total number of MCPs is 3 per stack $\times 2$ sets of stacks $\times 7$ polar angle channels, or 42 per TIDE sensor. The plates have pores of $25 \mu \mathrm{m}$ diameter, with plate thickness of $1.0 \mathrm{~mm}$. The ' $\mathrm{Z}$ ' stacks obtain a pulse height distribution with FWHM $\leq 60 \%$. Plate resistance's are in the range of $100 \mathrm{M} \Omega$ per plate. Flight plates are of 'imaging quality' without hot spots or noise enhancements, having passed a requirement of $\leq 10 \mathrm{~Hz} \mathrm{~cm}^{-2}$ at a gain of $1.5 \times 10^{7}$ at a threshold of $1 \times 10^{6}$ electrons at the output of the ' $Z$ ' stack. TIDE MCPs are handled under clean room conditions and TIDE is constructed of materials whose consistency 
TIDE Electron Optics Raytracing

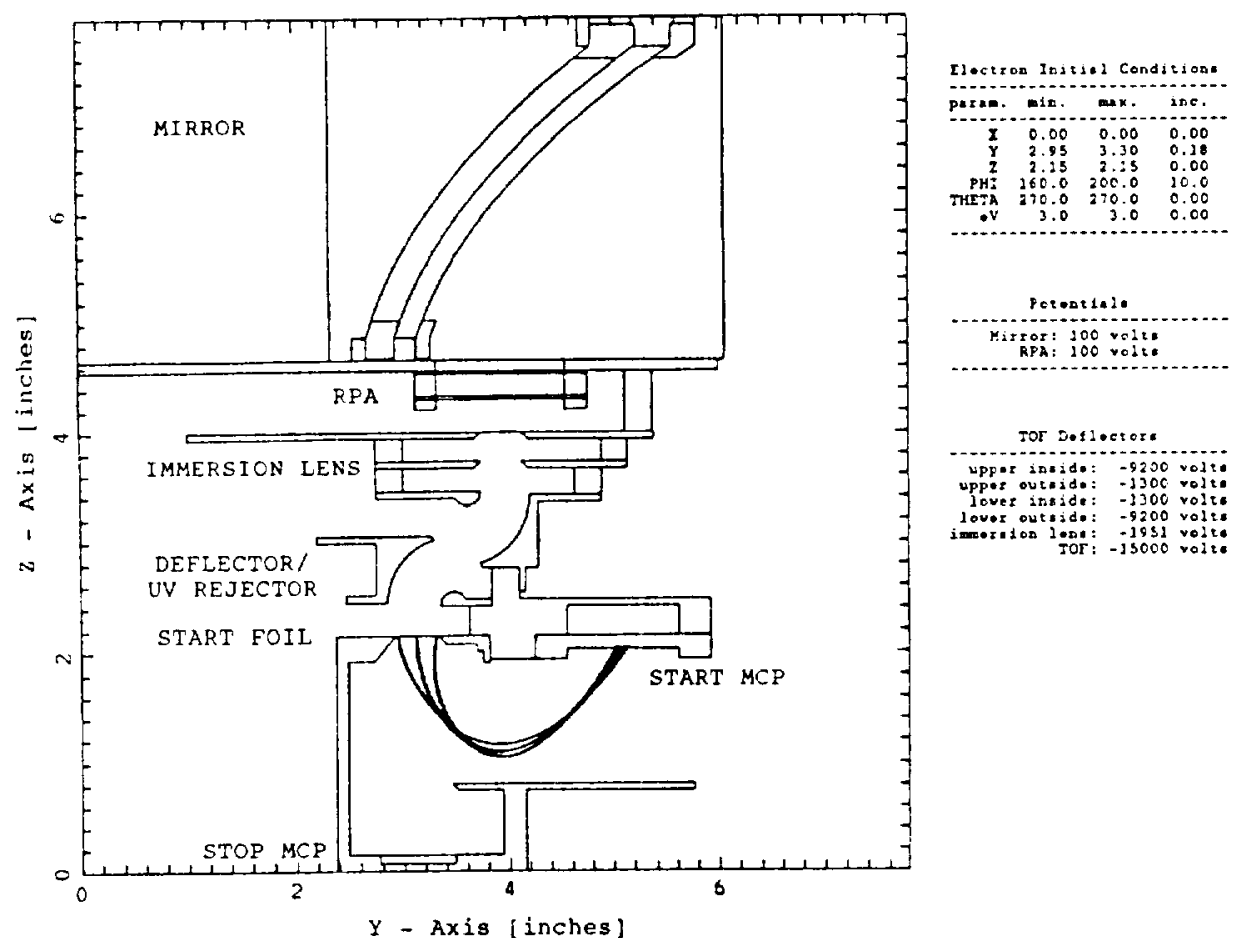

Fig. 7. Section view of the TIDE sensor similar to that in Figure 4, but illustrating the optics of collection of electrons emitted from the START foils by the START MCP detectors.

with long MCP life has been demonstrated by means of lifetime testing. They are burned in as stacks to $\geq 10^{10}$ counts $\mathrm{cm}^{-2}$ in a dedicated vacuum facility.

TOF LOGIC

All MCP pulses are counted without regard for the presence of a correlated pulse in another detector. These count rates are termed 'singles' rates to distinguish them from the more restricted set of correlated pulses resulting in time-of flight measurements.

In the ideal case every ion passing through the START foil creates one or more secondary electrons. These in turn are all collected and create a corresponding START pulse in the MCP. Every ion (or neutral) then proceeds from the foil to the STOP MCP and there creates a STOP pulse. (In fact, none of these events has a unity probability and the expected efficiency of the entire TOF event chain is $\sim 0.25$ ). In addition, there are random START and STOP signals due to penetrating radiation 
(in space) and to noise in the individual MCPs (field emission of electrons) giving rise to random coincidences. The possible cases may be summarized at follows:

\section{Start/Valid Stop}

In this case a 'start convert' pulse is generated and, at the conclusion of the process, a valid TOF value is also generated.

\section{Start/No Stop}

In this case the TOF circuit 'times-out' at a predetermined maximum time ( $300 \mathrm{~ns})$. The resulting TAC signal is not analyzed by the ADC because it contains no information and would only slow up processing. A 'time-out' signal is generated for each such event.

\section{Start/Second Start/Stop}

After the first START pulse the TOF circuit ignores any further START pulses that occur before either a STOP pulse or a 'time out'. Subsequent START pulses which occur during the $300 \mathrm{~ns}$ TOF window are not processed, and this time therefore serves as a (non-paralyzable) dead time for the circuit. Recall that the chance probability of a valid second START within the time-out window is small unless the count rate is extremely high, which would not ordinarily be permitted (see below).

\section{Stop/No Start}

The logic does not respond to a STOP pulse unless a valid START pulse has occurred within the previous period. By definition a START event is required to fire the logic.

\section{Start/Stop in Random Coincidence}

Nothing can be done about this possibility and a random TOF value is recorded. However, note that truly random coincidences are scattered uniformly across the TOF spectrum and this background can, in principle, be measured and subtracted. The expected rate for random coincidences is: $R_{12}=R_{1} R_{2} \tau$ where $R_{1}$ and $R_{2}$ are the START and STOP detector random rates, and $\tau$ is the TOF dead time of 300 ns. Taking $R_{1} \sim R_{2} \sim 100 \mathrm{~s}^{-1}$ for all seven START and STOP MCPs (which would correspond to relatively high background levels) gives:

$$
R=\left(10^{2}\right)\left(10^{2}\right)\left(3 \times 10^{-7}\right)=3 \times 10^{-3} \mathrm{~s}^{-1} .
$$

Thus the random rate should be relatively low, if the MCPs can be kept to background rates $\sim 10 \mathrm{~cm}^{2} \mathrm{~s}^{-1}$, a high upper limit except in the radiation belts. For a discussion of TIDE radiation shielding, see Moore and Young (1993). This also means that in the presence of a high flux of ions the random coincidence rate increases because it is proportional to either of the singles rates. 
During testing of the flight model, pickup of system noise by the STOP preamplifiers has been a chronic problem. This problem will be addressed during refurbishment by means of modifications of the grounding arrangement. Spurious STOP rates as high as $3 \mathrm{kHz}$ were observed during testing, but experience and the expression above agree that only very low random coincidence rates are produced at such levels.

\section{Scientific Data Types}

The TIDE TOF electronics produces four types of events which are counted and accumulated separately:

(1) Detector pulse 'singles' rates corresponding to the seven START channels and the one STOP channel.

(2) TOF logic 'singles' rates corresponding to 'Time-Out, 'Start-Convert,' and 'Reset' events. Since all valid coincidence events result in either a 'Start Convert' or a 'Time-Out', the 'Reset' rate is equal to the sum of their rates.

(3) 'Direct Events' or START/STOP coincidence pairs are accumulated into an array of 256 TOF bins by 7 polar-angle sector ID bins over $3 \mathrm{~min}$. without regard to energy step or spin azimuth sector.

(4) Coincidence pairs are also accumulated into an array of accumulators corresponding to 5 commandable TOF ranges (corresponding to the major species of interest), $32 \mathrm{E} / \mathrm{Q}$ bins, 7 polar-angle bins, and 32 spin azimuth bins.

\section{Dead Time}

Dead time corrections can be performed on the START Singles and the TOF data on-board by the TIDE DPU. The START Singles are subject to a $1.1 \mu \mathrm{s}$ dead time when not accompanied by valid coincidence event (START CONVERT) and to an additional $2.0 \mu \mathrm{s}$ dead time when accompanied by START CONVERT. Data associated with TOF events are always subject to the full $3.1 \mu \mathrm{s}$ dead time. The relevant dead times associated with various TIDE signals are given in Table III.

\section{PSI Description}

The PSI consists of the plasma source, a gas feed system with appropriate tanks, plumbing, and valving and an electronics system capable of powering and monitoring the operation of the PSI. The relationship among these components is illustrated in Figure 8.

The plasma source is a compact arrangement of a hollow-cathode, keeper and anode electrodes, a magnetic structure, and a grounded shield. Xenon gas flowing through the plasma source is ionized by bombardment with electrons released from a low-work-function surface within the hollow cathode. The ionized gas flows out of the plasma source, providing a medium-density $\left(\sim 10^{10} \mathrm{~cm}^{-3}\right)$, inert-gas plasma to neutralize differential charge buildup between various surfaces of the satellite 
TABLE III

TIDE live times and dead times

\begin{tabular}{lc}
\hline Signal & Time \\
\hline Start singles (w/o coincidence) & $2.1 \mu \mathrm{s}$ \\
Start singles (w/ coincidence) & $3.1 \mu \mathrm{s}$ \\
Resets (w/o coincidence) & $1.1 \mu \mathrm{s}$ \\
Resets (w/ coincidence) & $3.1 \mu \mathrm{s}$ \\
Timeouts & $3.1 \mu \mathrm{s}$ \\
Start converts & $3.1 \mu \mathrm{s}$ \\
Stops & $0.6 \mu \mathrm{s}$ \\
TOF, $M / Q$ values & $3.1 \mu \mathrm{s}$ \\
TOF direct event data & $10.1 \mu \mathrm{s}$ \\
Sample period (live fraction) & $5274 \mu \mathrm{s}$ \\
Sample period (dead fraction) & $586 \mu \mathrm{s}$ \\
\hline
\end{tabular}

Note: all values are preliminary and subject to further calibration and analysis.

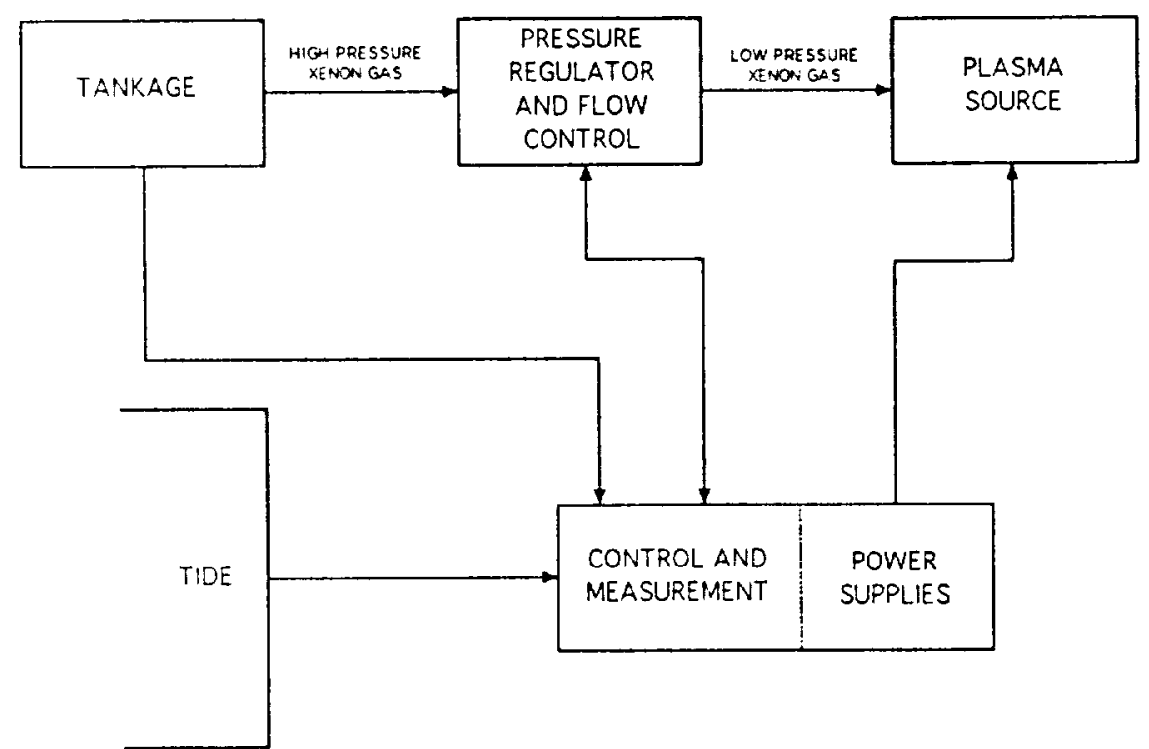

Fig. 8. PSI functional block diagram illustrating the relationships among the principal components

and also to form an electrically conducting 'bridge' between the satellite and the natural space plasma.

It is anticipated the plasma source will bias the satellite such that the plasma source anode is within a few volts of space potential. To hold the satellite within $+1 \mathrm{~V}$ of space potential it may be necessary to bias the plasma-source anode 


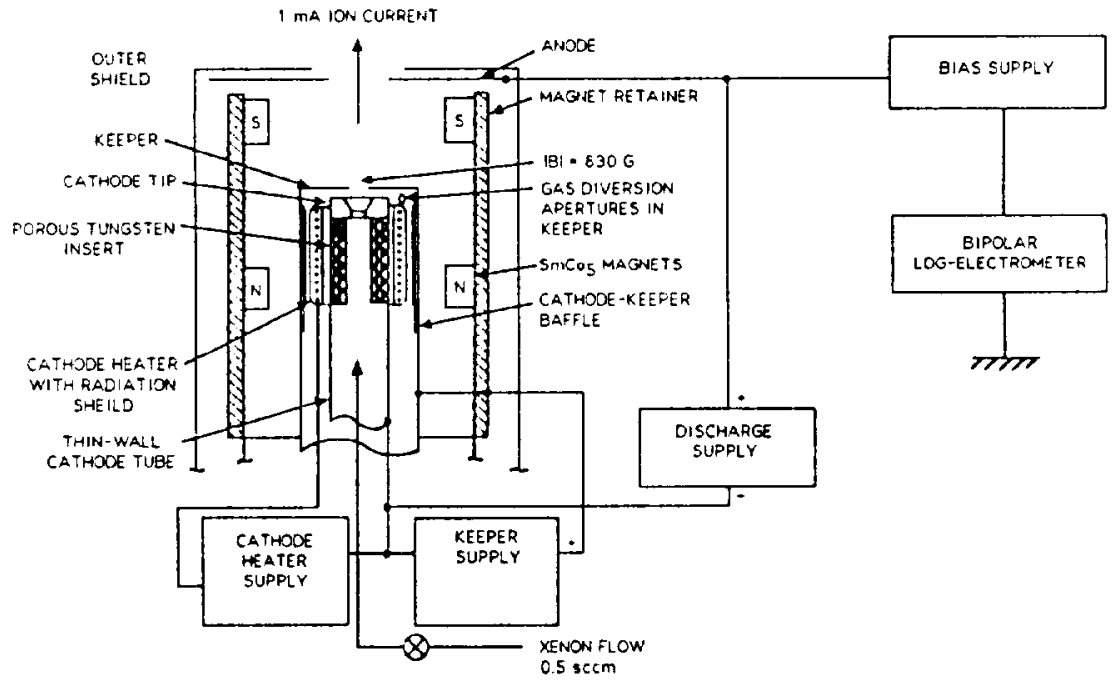

Fig. 9. Schematic illustration of PSI source, illustrating the roles of the several PSI power supplies in providing plasma emission and bias control.

relative to spacecraft ground. The PSI design provides a vernier bias control to permit this. The plasma source has the characteristics listed in Table II.

The plasma source has a porous-tungsten insert. The cathode, keeper, and anode are all electrically isolated from the outer can so that the return current from the satellite can be measured. The outer can is fabricated from cold rolled steel and thus serves as a magnetic shield for the permanent magnets inside the source. A solenoid valve in the gas feed plumbing is an additional source of stray magnetic fields. Both have been tested thoroughly, and their stray fields have been found acceptable.

\section{SOURCE SYSTEM}

The hollow-cathode source is illustrated schematically in Figure 9, and photographically in Plate 2 . The cathode is a porous-tungsten material impregnated with barium aluminate, a material with a low work function. Initially the cathode is heated with an external filament to begin thermionic emission. Xenon gas flowing through the hollow cathode is initially ionized by the thermionic electrons accelerated by the potential on the keeper electrode. The axial magnetic field serves to confine the electrons and increase the probability of an ionizing collision, thereby minimizing the rate of gas flow required to obtain a given ion current. Once the keeper discharge is initiated, a fraction of the positive ions is accelerated back to the cathode and produce local impact heating. After an initial heating time, the filament can be turned off and the thermionic emission is maintained without the penalty of heater power. 


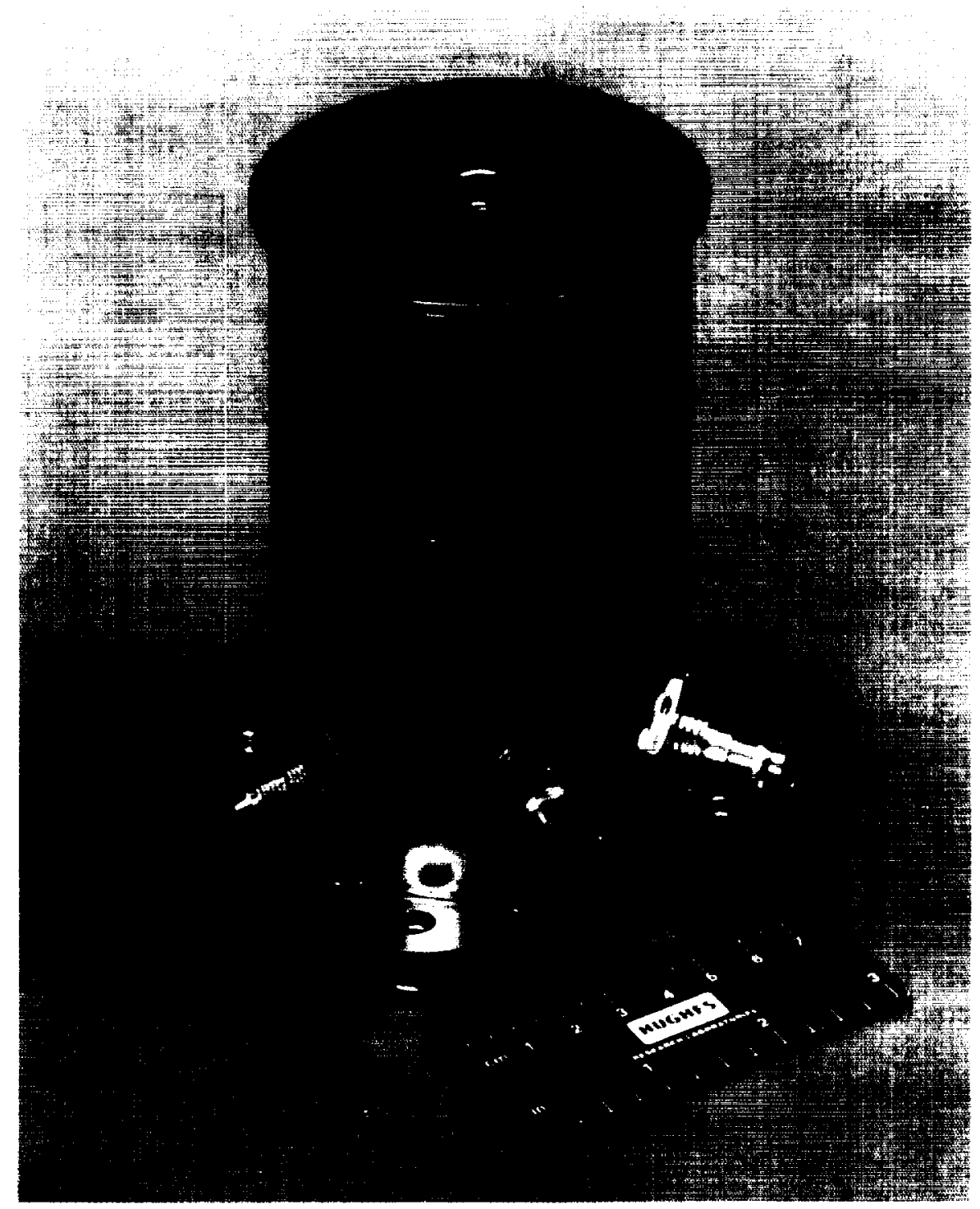

Plate 2. Photograph of the PSI source assembly. Plasma is emitted from the aperture at the end of the cylindrical package.

The keeper electrode and anode are biased +18 and $+25 \mathrm{~V}$, respectively, relative to the cathode. The neutral xenon gas flows throughout the entire region between the gas inlet and the outer shield exit. The potential structure accelerates electrons toward the anode, and production of plasma therefore occurs throughout the region. The distribution of plasma generation can be controlled to some extent by the magnitudes of the keeper and discharge voltages. A plume of low-energy plasma 
therefore exits the anode orifice, and the current can be 'fine-tuned' by adjusting the magnitude and polarity of the bias supply. This supply biases the entire plasma source relative to spacecraft frame ground, and an electrometer measures the net current produced by the source.

\section{PSI GAS FEED SYSTEM}

The feed system consists of the storage tanks, valves, pressure regulators, flow impedance, and pressure transducers required to provide the source with a steadystate 0.5 -std cm ${ }^{+3} \mathrm{~min}^{-1}$ flow rate. Its overall layout is illustrated in Figure 10 . The Xenon gas supply is contained in two pressure vessels located symmetrically on the spacecraft to maintain balance and provide gas flow over the anticipated range of temperature. The storage tanks are a pair of 4.81 , cylindrical high pressure vessels rated for $3100 \mathrm{psi}$. The tankage is fitted with high and low pressure transducers (to indicate the quantity of remaining expellant) and a manually operated fill valve. The nominal gas supply is $3.5 \mathrm{~kg}$, or 600 standard 1 , of Xenon, which is sufficient for $20000 \mathrm{hr}$ of continuous operation at the specified flow rate. The valves and regulators reduce the pressure to $10 \mathrm{psi}$ which is applied to the upstream side of a constant flow impedance plug to maintain a flow rate into the cathode of $0.5 \mathrm{std} \mathrm{cm}^{3} \mathrm{~min}^{-1}$, or 0.37 micro mole $\mathrm{s}^{-1}$. Saturation ion current is $1.0 \mathrm{~mA}$; hence, the maximum ion production efficiency is approximately $3 \%$.

\section{Operations}

TIDE

The performance of the TIDE/PSI system has been extensively simulated by 2.5 dimensional (i.e., axially-symmetric potentials) numerical raytracing computations and tested using a full scale prototype instrument. Significant iterations of the design were made and tested in this way, resulting in a thorough understanding of the electrostatic optics system. The final design has been explored with several million trajectories, resulting in a characterization of the angular and energy response over the full range of sensitivities for which the instrument can be programmed. The observed response of the instrument agrees well with the numerically computed response in most areas.

Figure 11 illustrates the simulated effective response width in energy, azimuth angle, polar angle, and effective area, as functions of the mirror ratio $R_{m}=$ $V_{m} / V_{\mathrm{RPA}}$. The response in spacecraft polar angle is essentially independent of mirror ratio. The spacecraft azimuth response is slowly varying, but does drop off by a significant factor at the low end of the total aperture range. The energy passband and the effective area Aeps each vary nearly a decade over the range plotted, reflecting the varying separation between the mirror and RPA energy cutoffs. At low mirror ratios, only the uppermost part of the mirror is active, where particle 


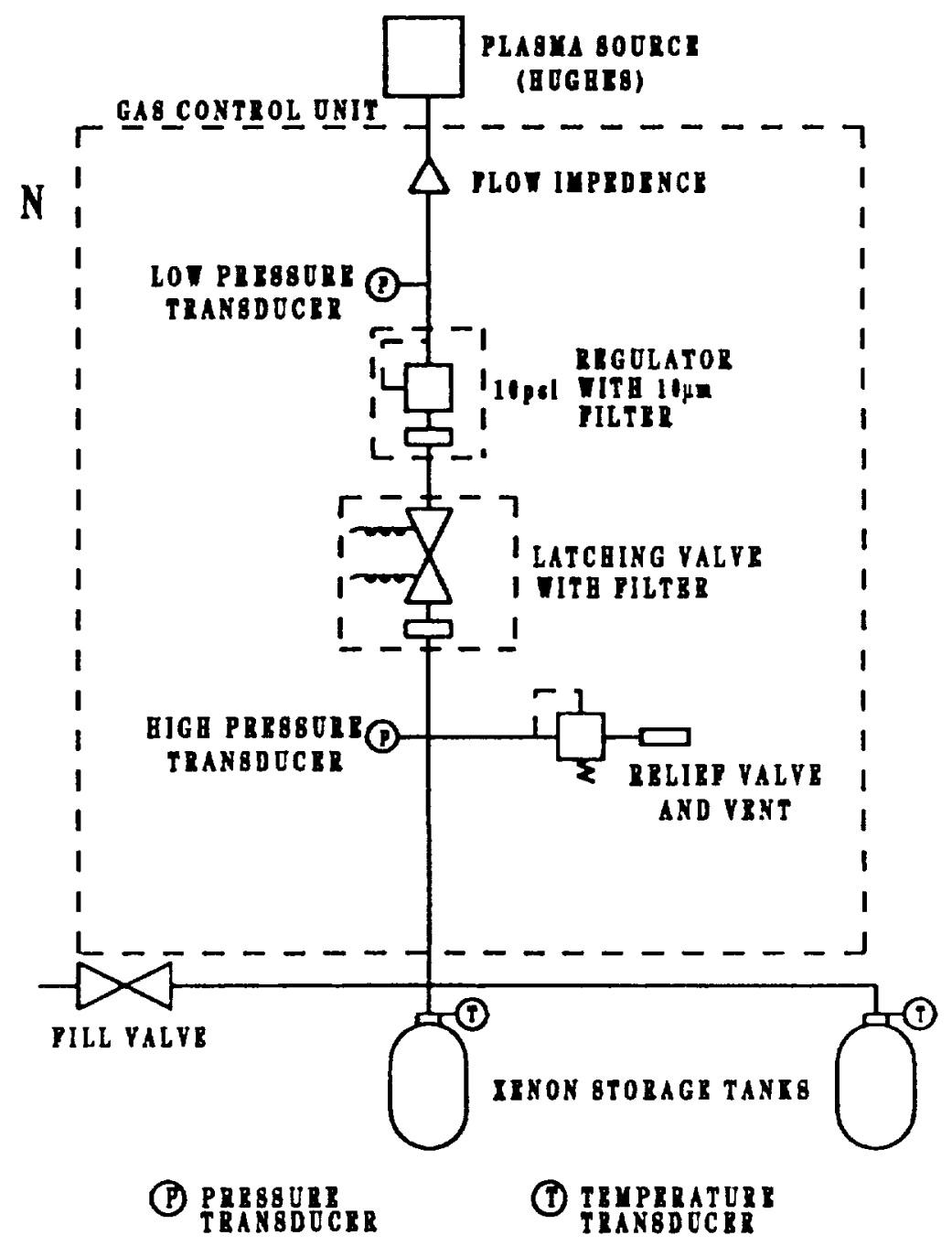

Fig. 10. PSI gas feed system block diagram.

incidence is more grazing. The simulation results make no accounting of grid losses, edge-effect losses, detection efficiencies for the foils or MCPs, or START-STOP coincidence probabilities and the effective area is therefore unlealistically high. End-to-end optics efficiencies are expected to be on the order of 10\% (applicable to non-mass-analyzed events on the START detectors), while the combination of foil conversion and START-STOP coincidence efficiencies is expected to introduce a similar multiplicative efficiency factor.

TIDE has been extensively tested and calibrated prior to delivery for integration on the POLAR spacecraft. Also shown in Figure 11 are the measured mirror ratio dependencies of the geometric factor and azimuth angle response width. An 


\section{TIDE Simulated and Measured Response}

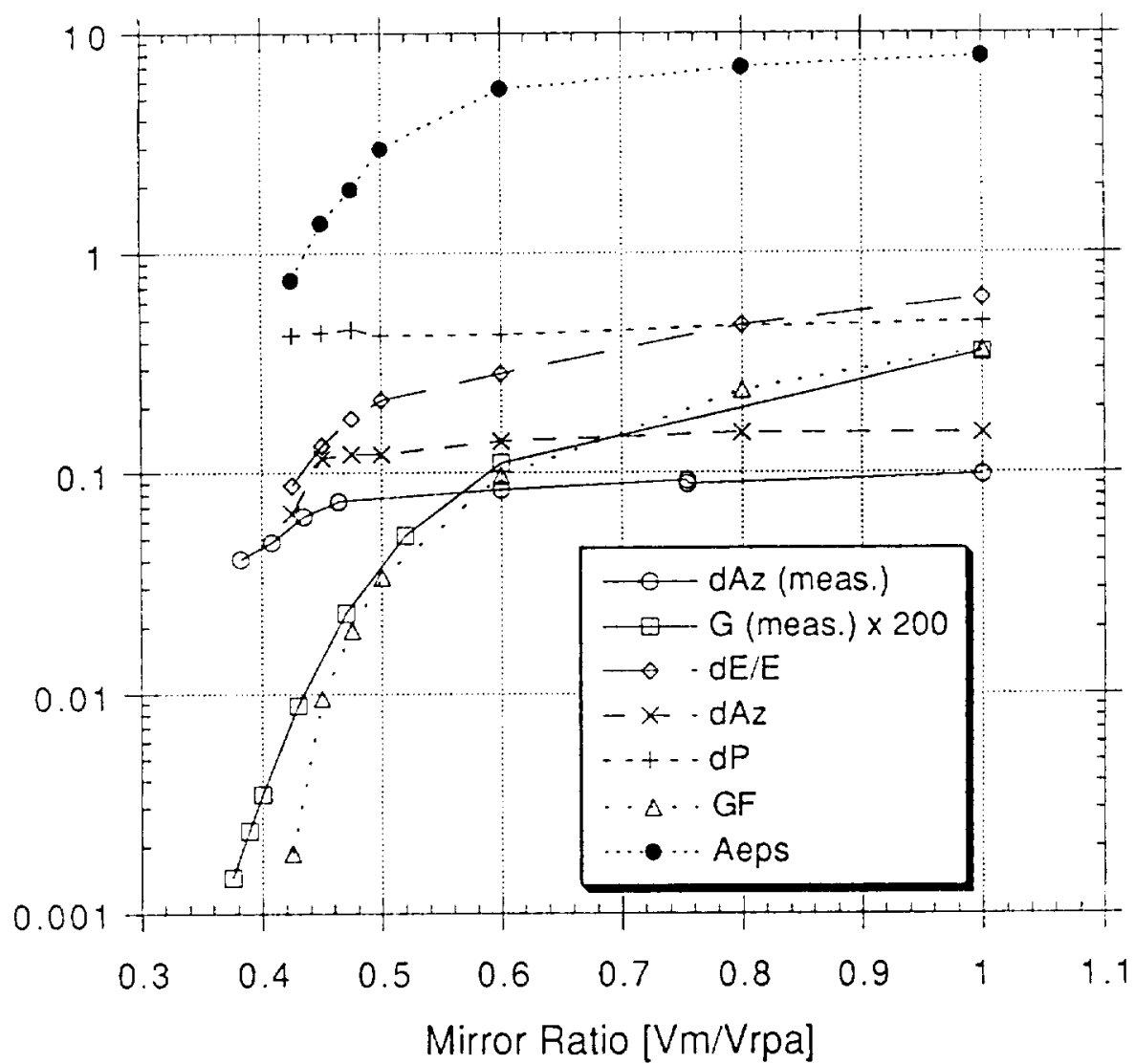

Fig. 11. The simulated and measured response of the TIDE instrument as functions of the ratio of Mirtor to RPA potentials. The geometric factor ( $\left.\mathrm{GF}\left[\mathrm{cm}^{2} \mathrm{sr} \mathrm{eV} / \mathrm{eV}\right]\right)$ is the product of the energy bandpass $(\mathrm{d} E / E)$, the azimuthal response ( $\mathrm{d} A z$ [radians]), the polar angle response ( $\mathrm{d} P$ [radians]), and the effective area $\left(\right.$ Aeps $\left.\left[\mathrm{cm}^{2}\right]\right) . G$ (meas.) is the observed geometric factor for TOF coincidence (mass-analyzed) events $\left[\mathrm{cm}^{2} \mathrm{sr} \mathrm{eV} / \mathrm{eV}\right]$, and $\mathrm{d} A z$ (meas.) is the observed effective width of the azimuth angle response (radians).

example of the data upon which these curves are based is shown in Figure 12. The calibration approach is to measure the response of the instrument to a monoenergetic and monodirectional ion beam with a cross-sectional area sufficient to completely illuminate one aperture. The geometric factor is then obtained from these data by integration. In Figure 12, the repsonse is plotted versus energy (or RPA voltage) and spacecraft azimuth angle, for two values of $R_{m}$. Maximum azimuth response is $\sim 6^{\circ}$, while the energy response width is $\sim 50 \%$ for $R_{m}=1$, and $25 \%$ for $R_{m}=0.6$. Polar angle response width (not shown) is $\sim 18^{\circ}$, independent of $R_{m}$. The measured response is generally in agreement with the simulations. A 
notable exception is the azimuth response, which is approximately $65 \%$ of its simulated value, owing most likely to slightly non-optimal potentials within the (UV) rejection/deflection system.

The calibration to date has not been definitive with regard to absolute effective area and geometric factor, for the following reasons: First, the MCPs used during the initial calibration were engineering plates with long test histories. Second, the test setup was designed for angular response scanning and the beam current measurement was located somewhat upstream of the entrance aperture. Based upon the simulations and the data in hand, it is anticipated that the non-mass analyzed effective area will be $\sim 1 \mathrm{~cm}^{2}$, and that the mass-analyzed effective area will be $\sim 0.1 \mathrm{~cm}^{2}$.

Figure 13 illustrates a TIDE mass spectrum constructed by superposition of spectra from tests done with the beam source dominated by four different species, simulating a multi-constituent ion beam. The main point here is to illustrate the modest mass resolution for which TIDE has been designed in order to maximize its overall sensitivity to the major ion species known to be of interest in the terrestrial magnetosphere and ionosphere. (Note: In these tests, the TOF analysis was suffering from an electronic problem which both reduces the overall signal/noise and introduces artifactual peaks in the range of Channel 1-50. It is believed, based on testing with engineering model equipment, that this problem has been largely corrected. Operations during refurbishment and on orbit are expected to reflect significantly improved performance in this area.)

The instrument response function has been used to compute the expected instrument response to hypothetical drifting bi-Maxwellian distribution functions with parameters covering the full range of those expected during flight operations. This procedure provides an end-to-end test for the flight and ground software which is used to store, process, and analyze the TIDE data, as well as a means of designing operating modes intended to resolve special features of the data.

Of particular interest is the evaluation of the adequacy of TIDE to resolve the features of interest in the very high altitude plasma, while maintaining the capability to observe the denser plasma of the inner magnetosphere. Figure 14 illustrates the results for two cases of interest, including a very high Mach number polar wind case and a hot upwelling ion (bi-Maxwellian) case. It may be seen that distribution functions deduced from the TIDE data are expected to be both qualitatively recognizable, as well as quantitatively accurate.

\section{PSI}

The plasma source component of TIDE/PSI has required less development than TIDE itself, being a direct copy of the plasma source device developed for the USAF by Hughes. Moreover, its operation is essentially passive in that it reacts very weakly to its environment and is self-regulating in terms of the equilibrium floating potential of the spacecraft. Nevertheless, there are significant choices to 


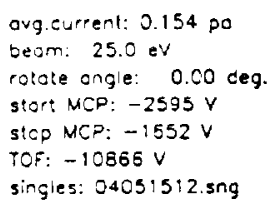

TIOE Col (CTP;6.2.6) Ch.3 Angle Response

April 5, 1993, 15:14 to 15:30

$v_{\text {mir }} / v_{\text {rpo }}=0.6$

RPA: $10.0-40.0 \mathrm{~V}$ exponential

Start Singles, Channel 3 eontour limits

in $\log _{10}($ her:i $)$

$\min =1.0$

$\operatorname{mox}=60$

inc $=0.25$

energy: ev25e:2.e

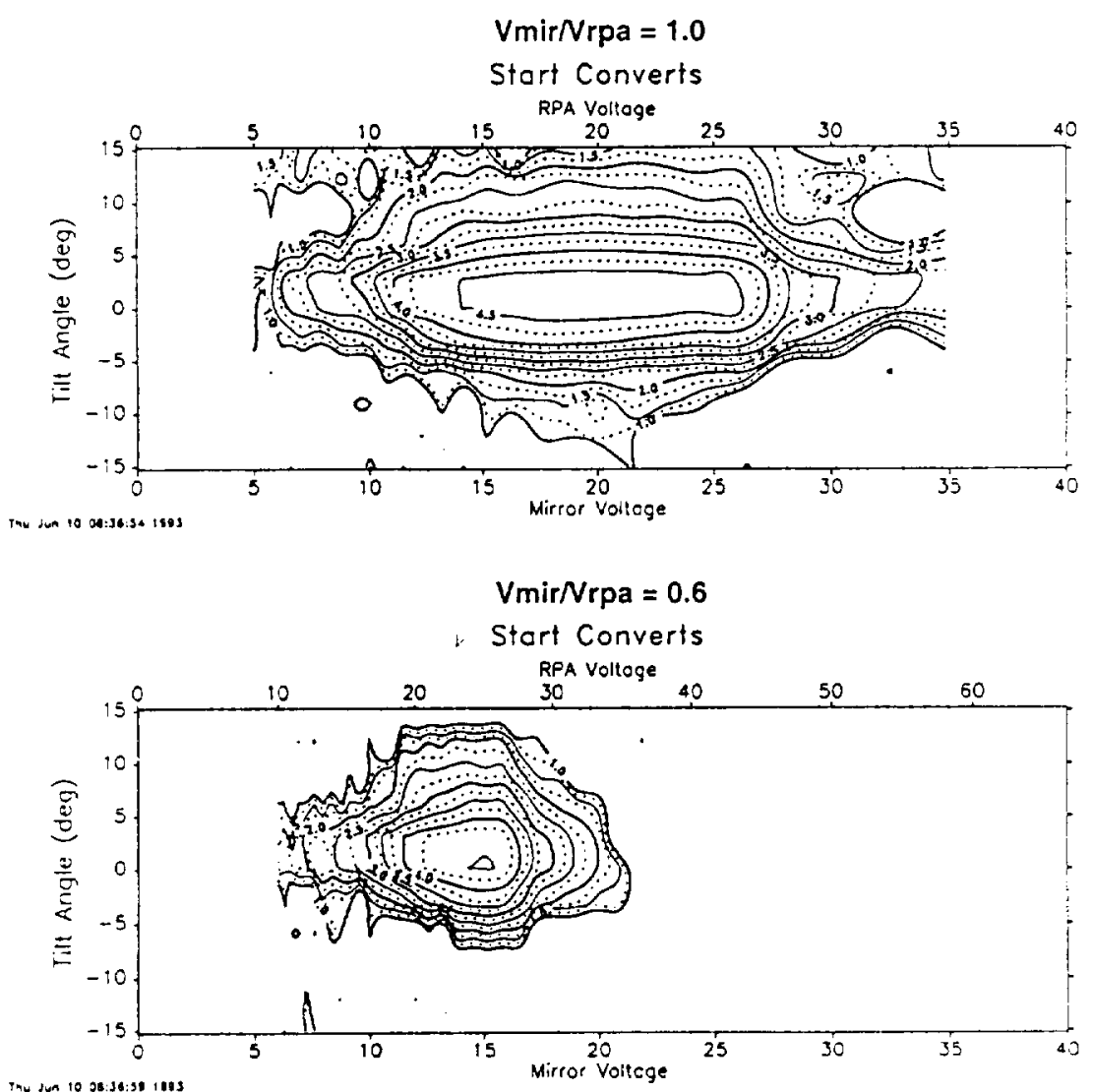

Fig. 12. Measured response of a TIDE aperture versus RPA voltage and azimuth angle, for a $25 \mathrm{eV}$ beam.

be made in operating the PSI, in terms of the potentials and internal currents which are set so as to provide optimal performance. In general, there is a tradeoff between stability of the PSI discharge and its emission capability. Since the current requirements for the POLAR spacecraft (tens of $\mu \mathrm{A}$ ) are far below the capabilities of the plasma source (>1 mA), its operating point is chosen so as to strongly favor stable operations (i.e., without fluctuations of the plasma output) at the expense of maximum emission capability.

To illustrate this point, we provide a plot of the operating parameter space for PSI in Figure 15, based on laboratory tests. The design operating point is indicated, 


\section{TIDE Calibration: Summed Spectra $\mathrm{H}_{2}$, $\mathrm{He}, \mathrm{Ne}, \mathrm{N}_{2}$ gas bleeds}

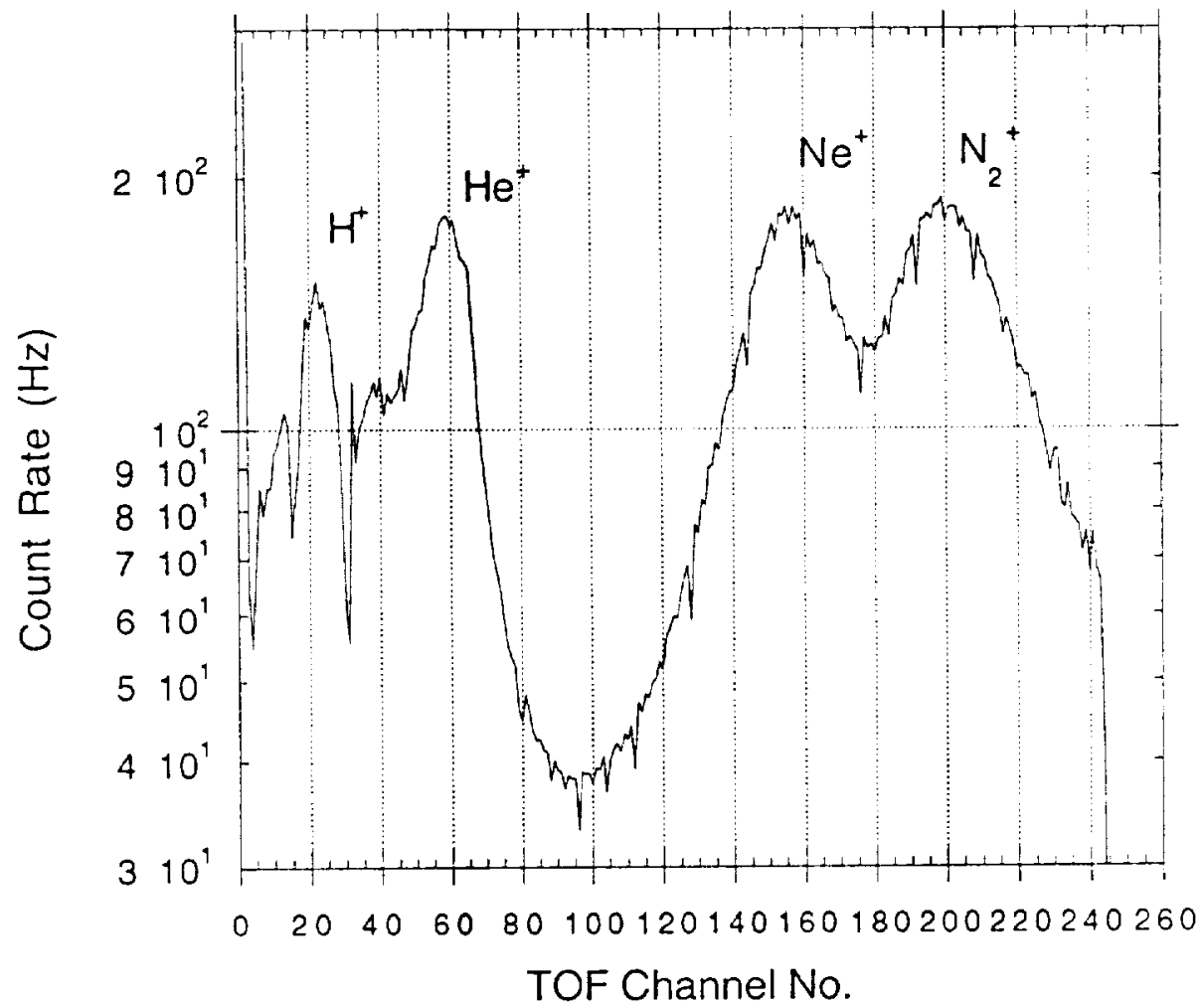

Fig. 13. TIDE mass spectral response formed by superposing the response to laboratory beams generated from four different gas leaks.

as well as a wide range of parameters which would produce considerably larger plasma output in some regions at the expense of smooth and stable operation.

\section{MEASUREMENT MODES}

With TIDE's capability to monitor multiple directions and mass species simultaneously, only the ion energy needs to be swept. Since TIDE has only one swept measurement, all its operating modes consist of repetitive sweeping of the mirror/RPA potentials in an uplinkable pattern coordinated with the spin sweep of the seven sectors in spacecraft azimuth with a period of $6 \mathrm{~s}$. TIDE has two sampling modes. The 'standard' sampling mode provides for 32 energy steps per sweep with 32 sweeps per 6 -s spin, dividing the spin into $11.25^{\circ}$ sectors. A phase-locked-loop maintains precise spin sectoring during each spin, and provides for the phase to be adjusted by up to $22.5^{\circ}$ of spin in 256 steps. 

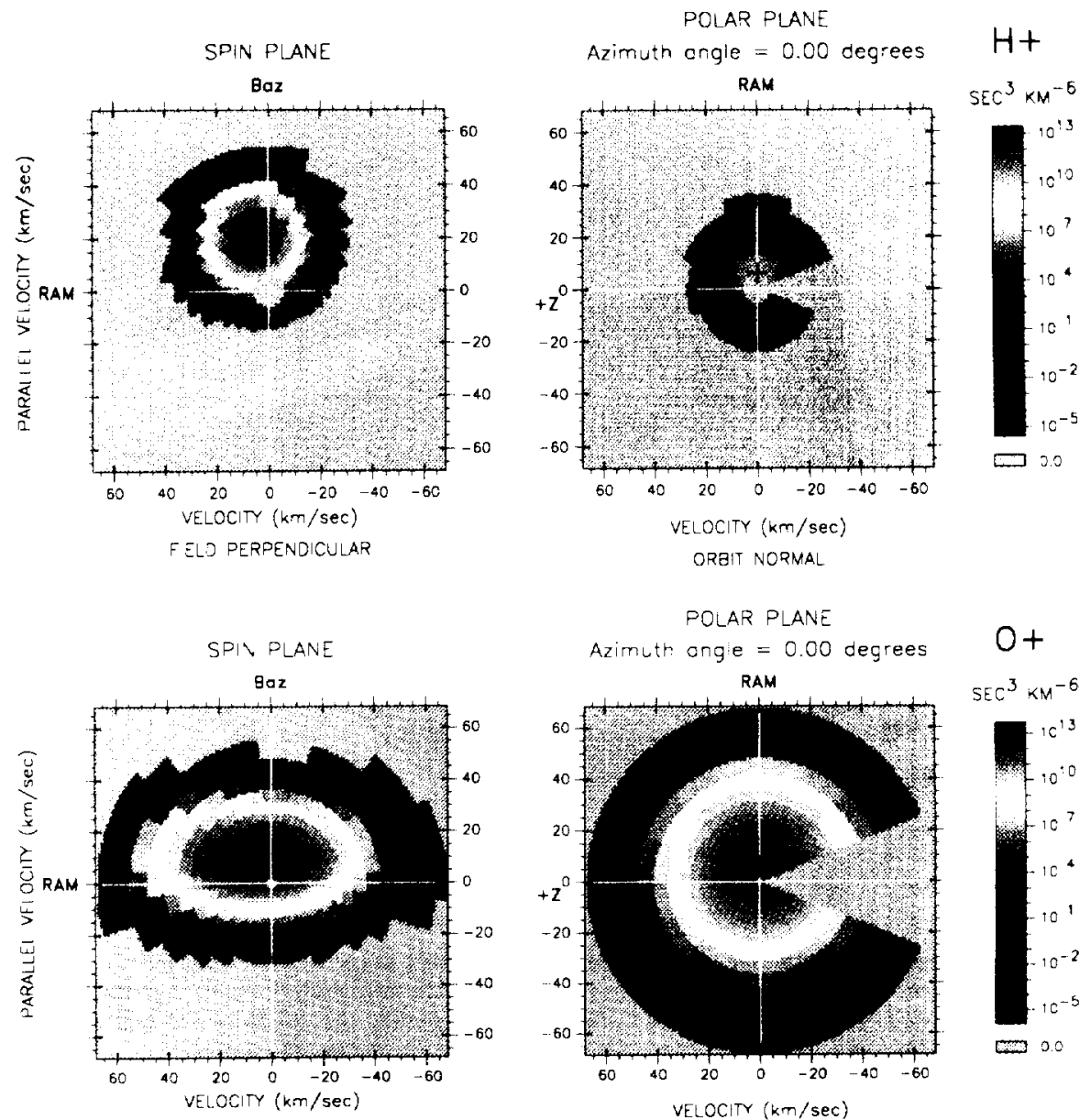

Fig. 14. Unsmoothed TIDE response to a polar wind distribunon (upper panel: $V_{\|}=21 \mathrm{~km} \mathrm{~s}^{-1}$, $T=0.15 \mathrm{eV}$ ) and to a bi-Maxwellian model of an upwelling ion distribution (lower panel: $T_{\|}=3 \mathrm{eV}$, $T_{\perp}$ ). Sampling resolution corresponds to the normal operating mode for TIDE inflight.

The fundamental organization of the TIDE data consists of a triplet of arrays: First, the species array has dimensions: energy, azimuth angle, polar angle, and mass, with 16 bits of accumulation depth. Second, the singles array has a similar structure, but with four additional rates in addition to sectors 1-7: STOPS, StartConverts, TimeOuts, and Resets. Finally, the direct event array stores full mass spectral information (8-bit address) for each of the 7 sectors, without regard to spin or energy, and with 32 bits of depth to accommodate accumulations of durations up to several minutes.

The data reside in the spin data acquisition memory which includes two pairs of identical banks ( $64 \mathrm{k} \times 16$ bits each) that collect data during alternating spins for the species data and singles/direct events, respectively. While data are being 


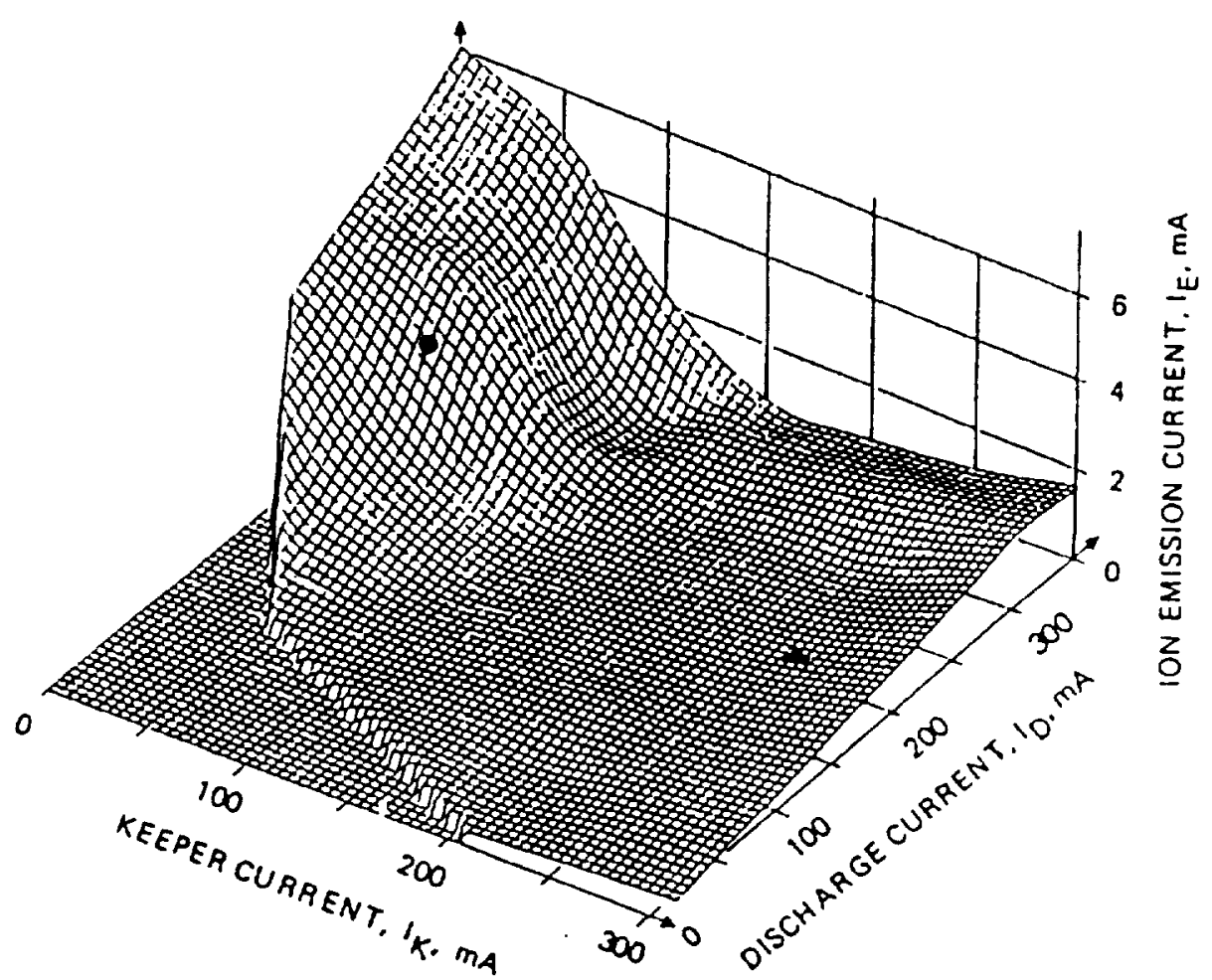

Fig. 15. PSI operating parameter space illustrating the wide range of operating points which are possible. Selection of an operating point in flight is based upon minimum fluctuation in the discharge and emitted plasma current. The triangle indicates approximately the conditions at which PSI will operate on POLAR, while the circle indicates a high-current capability alternative.

acquired in one pair of memory banks, processing is performed on previously acquired data, resident in the alternate pair of memory banks. During processing, specified data sectors can be copied, summed, or differenced with selected portions of bulk dynamic RAM memory ( $384 \mathrm{kB}$ ) that serves as the main working memory of the Data Processor. A schematic showing the basic functional elements of the TIDE data flow is shown in Figure 16.

In view of the large size of the TIDE data 'images' (Species: 32 energies $\times$ 32 azimuths $\times 7$ polar-angle channels $\times 5$ masses $\times 16$ bits $=70 \mathrm{kB}$, Singles: $11 \times 32 \times 32 \times 16$ bits $=22 \mathrm{kB}$, and Direct Events: $256 \times 7 \times 32$ bits $=7 \mathrm{kB}$; Total: $99 \mathrm{kB}$ ), and the rate of generation of images (once per 6-s spin), the intrinsic data output of TIDE may be seen to be $\sim 10^{5}$ Bytes $\operatorname{spin}^{-1}$ or $\sim 130 \mathrm{kbps}$. The allocated telemetry rate is 3000 Bytes spin ${ }^{-1}$ or $4000 \mathrm{bps}$, so it is clear that tradeoffs must be made between temporal resolution and comprehensiveness of data reporting. TIDE has two data handling modes, which provide considerable flexibility for trades in favor of the one or another aspect of the data set. In addition, onboard 


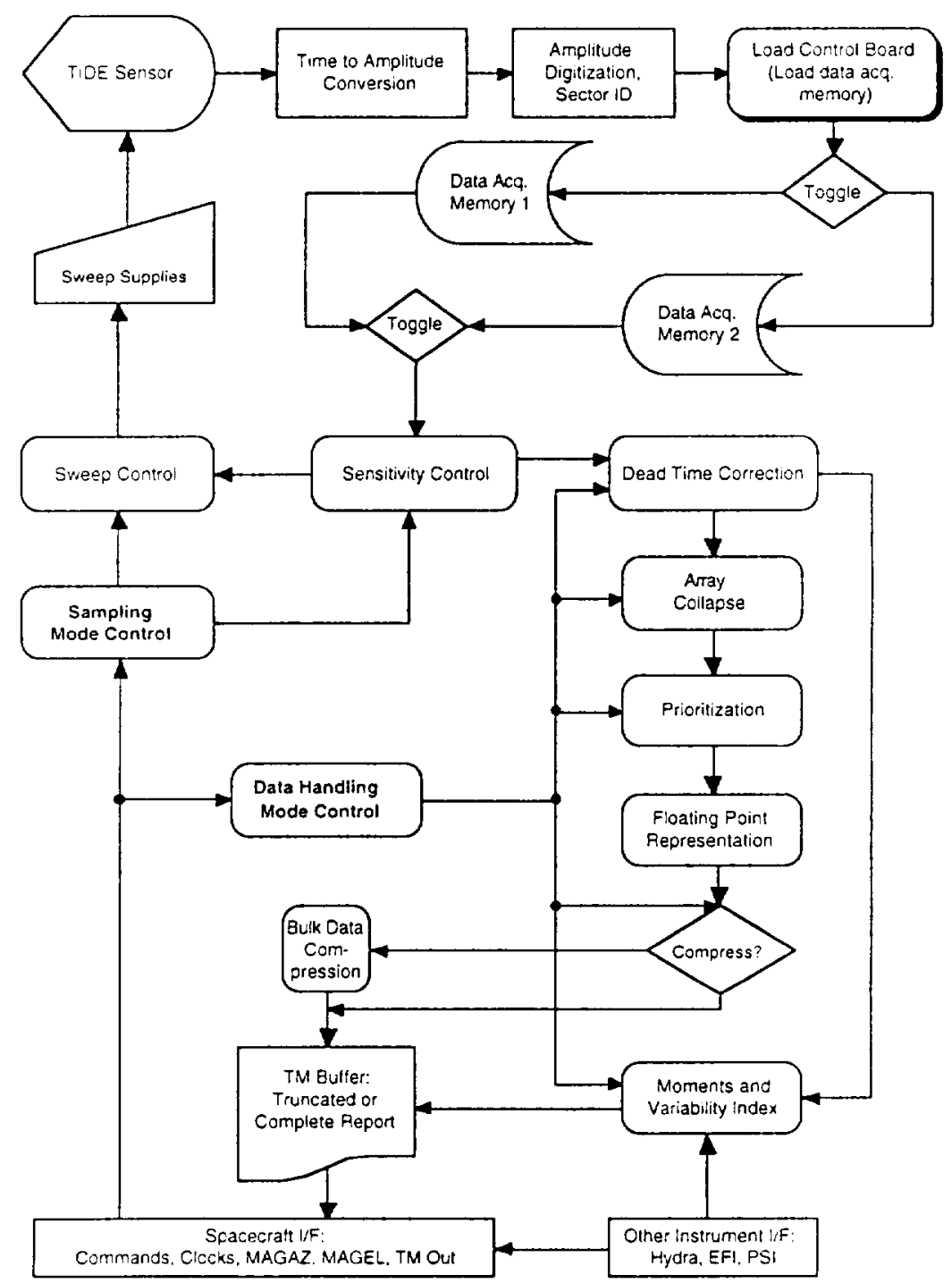

Fig. 16. TIDE dataflow chart.

data processing techniques such as data compression and moment computation are used to maximize the effectiveness of the available data rate.

\section{Data Decimation}

The following methods implement the necessary trades by reducing the content of one or another aspect of the data to be telemetered:

Floating Point Representation. TIDE count accumulations are reduced from 16 
bits to 8 bits (or 32 bits to 16 bits in the case of direct event data) by implementing a special form of floating point representation with a resolution of $\sim 3 \%$ and a range of $2^{16}$, conserving scarce telemetry and limiting the reporting precision to a level commensurate with the overall measurement accuracy. The mapping of 16-bit internal data acquisition accumulator words to encoded bytes is handled in hardware by a memory lookup table.

Azimuth Collapse. To make the angular sampling which occurs near the spin axis directions in the TIDE sampling scheme more commensurate with the angular response of the instrument, data from the polar angle sectors can be increasingly collapsed as they approach the spacecraft spin axis. This yields a collapse factor of 1.9 without significant loss of detail in the data. To provide additional collapse at the cost of real loss of angular resolution, an additional collapse option combines alternate bins for a collapse factor of 3.8. Another collapse option simply combines 32 spin rows into two rows for laboratory test and calibration purposes where spin sectoring is not needed. Only the second of the two spin rows is reported, so that the first half of each 'spin' period may be used to make adjustments to the laboratory setup in real time without invalidating the entire spin of data.

Energy Array Collapse. In cases where additional collapse is desired, an energy collapse is provided in which adjacent energy bins of data are combined, for a collapse factor of 2 .

Priority Ordering. The first form of prioritization of the TIDE data is the drawing of a distinction between the reporting of species data and direct events. Since the purpose of direct event reporting is to provide information about species which may not be sampled among the top priority species data, it is intrinsically of lower priority. It is therefore accumulated over a longer time scale of 32 spins (a superspin, see below). This requires larger accumulation registers, but drastically reduces the data rate requirement for this information, down to 56 words (long 4 Byte) per spin (of 3000).

The TIDE species data are also routinely priority ordered from the most important species to least important species. The ordering is controlled by a default table in ROM, but may alternatively be controlled by means of an uplinked table. The START singles rates (seven of them) may be treated as a distinct, composite mass species and introduced into the telemetry as such. By default, the START singles data is the highest priority mass species in the priority ordering. The other four singles rates, STOPS, StartConverts, TimeOuts, and Resets, are reported only after reduction to spin moments, as described below.

\section{In-Flight Data Processing}

In order to minimize the impact of data decimation, the following in-flight processing techniques are used: 
Bulk Data Compression. Collapsed arrays of floating point represented data are further compressed by a lossless encoding technique similar to those used for telecommunications of computer files. This eliminates data with little information content and provides reductions in the total data volume by a factor ranging from approximately 2 to several, depending on the information content of the specific data. The resulting data volume depends in general upon the nature of the data collected, but this uncertainty is handled by truncation or by allowing as much time as is needed for reporting. The result is the transmission of more data content per spin (less truncation), or a reduced number of spins between complete reports, respectively.

Moment. Moments computed each spin provide an important measure of variability from spin to spin when full distributions can only be sent at less frequent intervals. In addition, moments of the singles rates provide useful diagnostic information concerning the operation of the instrument detectors and electronics.

Selected simple moments of the species and singles, angle and energy distributions, are computed from the raw data arrays and reported on a single spin basis. A short history of these moments is updated by the Data Processor each spin and used to judge the variability of the data for purposes of triggering a high time resolution mode. The criterion for the switch is that the standard deviation within the current record of selected moments exceeds an uplinkable threshold.

Owing to limited computational speed, even with two 32-bit processors, the moments computed are total events, simple mean Azimuth and Polar Angle bin numbers, and effective widths for Azimuth and Polar Angle. The energy distribution is weighted to provide the energy of maximum phase space density and the effective width of the phase space density distribution. It is, of course, planned to develop the full physical moments from the downlinked data.

Dead-Time Correction. The finite dead time of the TOF analysis circuits, and the desirability of operating with large counting rates to maximize the statistical significance of the TIDE data, indicate that TIDE requires a dead-time correction onboard to support the computation of accurate collapses and moments. Therefore, it is necessary to perform an onboard dead-time correction of all accumulations based upon the overall rate of TOF events. This is done on the basis of the current START CONVERT rate, by means of a look up table stored in DPU memory.

\section{Operating Modes}

Operation and Commanding. The strategies for reducing TIDE data volume involve a number of options, the selection of which defines a TIDE operating mode. In addition, the basic partitioning of energy and angle sampling can be modified so as to gain fine angular resolution at the cost of reduced time resolution. The mode structure is summarized in Table IV. In the following paragraphs, the major 
TABLE IV

TIDE operating mode summary

\begin{tabular}{|c|c|c|c|c|}
\hline $\begin{array}{l}\text { Mode } \\
\text { nomenclature }\end{array}$ & $\begin{array}{l}\text { Energy } \\
\text { bins }\end{array}$ & $\begin{array}{l}\text { Azimuth" } \\
\text { bins }\end{array}$ & $\begin{array}{l}\text { Mass } \\
\text { bins }\end{array}$ & Comments \\
\hline \multicolumn{5}{|l|}{ 'Standard' sampling } \\
\hline Calibration collapse & 32 & $1,1,1,1$ & 5 & collapse out spin information \\
\hline Truncate (high time res.) & 16 & $16,8,4,2$ & 3 & maximum collapse \\
\hline Complete & $16-32$ & $32,16,8,4$ & $1-5$ & on trigger $\rightarrow$ truncate \\
\hline
\end{tabular}

High ang. res. sampling

$\begin{array}{lllll}\text { Truncate } w / \text { phase stepping } & 16 & (32,16,8,4) \times 4 & 3 & \begin{array}{l}\text { step hase by } 2.91 \text { deg spin }^{-1} \\ \text { Complete } w / \text { phase stepping? }\end{array} \\ 16-32 & (32,16,8,4) \times 4 & 1-5 & \text { on trigger } \rightarrow \text { truncate }\end{array}$

"Note: The series of numbers under 'Azimuth bins' corresponds to the number of azimuth bins in polar angle sectors 4 and 5,3 and 6,2 and 7, and 1, respectively, i.e., in sectors ranging from the spin plane to the spin axes.

mode options are described.

Calibration Mode. During testing and calibration on the ground, there is no 'spin' dependence of the data. A 'calibration mode' has been developed for use in this context. In this mode, the normal 32 spin sectors are summed into two sectors, one of which is discarded to allow for settling of changes made in the test setup, while the latter of the two is reported. This reduces the data volume by a factor of 32 , removing over- sampling that is inappropriate at the time of calibration, and making it possible for all of the collected data to be reported within a single 'spin,' as defined by the simulated spacecraft spin clock provided by the GSE.

In addition, a unique additional data product is defined for this mode containing all singles rate data, which is plotted in real time to facilitate management of detector and TOF high voltages during testing.

Truncate (High Time Resolution) Mode. Data are truncated to the amount which can be sent in a single spin of telemetry, but with priority ordering to insure that the most important data are sent. To maximize the number of species reported on in this mode, maximum collapses are used for both energy and azimuth angle. Since each species requires approximately 940 Bytes at this level of collapse, while 3000 Bytes can be telemetered per spin, only the three highest priority species can be monitored in this mode. By default, the three species reported are START singles, Mass $1\left(\mathrm{H}^{+}\right)$, Mass $2\left(\mathrm{He}^{++}\right)$. However, this designation can be modified by uplink of an alternate priority table. 
Complete Report Mode. In order to report a larger number of mass species or to regain the full available resolution in energy and azimuth, or both, it is necessary to give up time resolution and report the full data set over a period of multiple spins. The full ordered data array is queued for telemetry, in general leaving a partial spin of telemetry unfilled. In order to fully utilize the available telemetry, the next spin header is introduced at mid-spin followed by the next spin record without waiting for the next spin clock pulse. This is possible because of the buffered nature of the data processing. The mean number of spins required to report a data array is, to a large extent, controlled by the level of collapse which is implemented and the character of the data itself.

The Complete Report mode operations result in a time series of data arrays for a selectable number of species (from 1 to 5 plus START singles), at time intervals ranging from every spin to every several spins, depending on the collapse level. Moments of the data, computed and reported every spin, may be used to identify the existence of variability on time scales shorter than the current number of spins between distribution samples (see below).

TIDE monitors the EFI (electric field investigation) and Hydra (three dimensional energetic plasma) experiments' 'burst' modes by means of dedicated lines connecting the instruments. It also monitors the reduced moment data from the most recent several spins of data. TIDE can be enabled to switch to its high time resolution mode, truncating data to permit single spin reporting, when a specified combination of these sources indicates the presence of variability in the plasma environment meeting criteria which can be defined by uplink. It dwells in the high time resolution mode for an uplinkable duration.

High Angular Resolution Mode. As noted above, the 'standard' sampling is based on 32 energy sweeps of $11.25^{\circ}$ duration each. The M/RPA optics supports a finer sampling of Spin Azimuth angle when operated at reduced sensitivity. The ultimate angular resolution of the optics is limited by the available flux, but it is thought that resolutions down to approximately $3^{\circ}$ can be supported.

A 'high angular resolution' sampling mode is based on the capability to vary the sector phase from spin to spin. By stepping the phase of the sectors through a submultiple $(N)$ of $11.25^{\circ}$ each spin for $N$ spin reports (plus another spin to restore the phase to baseline), a factor of $\mathrm{N}$ finer angle sampling is supported at the cost of a loss of time resolution by a slightly larger factor. The value of $N$ commensurate with maximum practical angular resolution is approximately 4 . The spin phase is incremented once per telemetry report so that both truncated and complete reporting are supported by this mode.

Over Counting and Auto Sensitivity Control. The basic strategy involves sampling the entire data acquisition memory and counting the number of saturated counting values which exceed a hierarchy of thresholds. For each energy (RPA) step of the current spin of data, if a significant number of samples exceed a particular 
threshold in the hierarchy, the sensitivity is reduced by a corresponding number of 'stops', where the term 'stop' is used by analogy with mechanical aperture systems. The next spin of data is again tested for saturation as described above and further reduction is imposed if needed, continuing until the over counting criterion is no longer met.

The flight software provides 16 sensitivity 'stops'. These are set at equi-log intervals in geometric factor, across over three decades, based on the measured variation of geometric factor with mirror ratio (see Figure 11). The lowest two 'stops' each correspond to a complete shut-down of the instrument, first at a mirror ratio of 0.3 , then at a ratio of 0.0 .

When a low counting threshold is exceeded by an insufficient number of samples, the sensitivity can be increased, at a maximum rate of one 'stop' per spin. The spacing between thresholds and 'stops' is determined from laboratory testing of the control algorithm. The overall control strategy is best described by an analogy with the human eye. Upon encountering a bright light the eye blinks and then slowly recovers by squinting and slow reopening.

The testing process is repeated and implemented every spin. More elaborate recovery algorithms using the number of saturated counting values from previous spins in a linear scaling procedure could also be implemented if deemed necessary. However they would require additional coding and control functions, and thus they are not implemented in the baseline software scheme.

TIDE sensitivity control is implemented in flight software at two levels. At the default level, over counting in a particular energy step at any point during a spin triggers a proportionate reduction of the sensitivity level for that step in the energy sweep. Cessation of over counting does not, however result in an increase in the sensitivity for that energy step, and the instrument becomes adjusted to the largest fluxes encountered at each given energy step of the sweep. At the optional level, the instrument responds to low counting levels by returning the sensitivity to higher values at each energy step, at a maximum rate of one 'stop' per spin.

Default sensitivity control is in force at all times when the instrument is not being monitored in real time, to protect against over counting. The lowest available sensitivity 'stop' is designed to totally shut down the flux to the detection system. In the event that this still fails to eliminate over counting on any given energy step, the control software causes shutdown of the High Voltage supplies, TOF first, then MCP bias. The default sensitivity control can be disabled by means of an appropriate command, which is designated as hazardous.

\section{Science Operations}

The various data products generated by TIDE/PSI are summarized in Table V. TIDE Science Operations will consist primary of the proper selection of modes controlling the properties of these data products. To support the ammassment of a large synoptic data set, TIDE is designed insofar as is possible to make observations in a constant and repetitive fashion, with a minimum of mode switching. 
TABLE $V$

Summary of TIDE/PSI data products

\begin{tabular}{ll}
\hline Data product & Supports derivation of \\
\hline Every spin & Key parameters moments \\
(for each species) & Ram flow \\
Mean energy & Temperature \\
Energy width & Flux \\
Peak count rate & Vertical flow \\
Mean azimuth & Temperature \\
Azimuth width & Cross flow \\
Mean polar angle & Temperature \\
Polar angle width & Definitive moments \\
Every $\sim 2-4$ spin depending on & \\
compression: (Full image for each species) & Density, temperatures (perp. and parallel), \\
Distribution array & flow velocity (3D), heat fluxes, \\
32 energies ( $0.1-500$ eV) $\times 32$ azimuths & superthermal features identification \\
$\left(11.25^{\circ}\right.$ bins) $\times 7$ polar angles $\left(22.5^{\circ}\right.$ bins) & Very high Mach number flow temperatures \\
High angular resolution supports $2.8^{\circ}$ & \\
azimuth bins with $4 \times$ longer accumulations & Single spin spatial resolution for full \\
High time resolution for major species by & distribution arrays, limited species \\
truncation with priority ordering & Minor ion species abundances \\
Every superspin $(32$ spins) & Optional promotion to major species \\
Accumulated mass spectrum & Optional promotion to major species \\
128 mass channels $(1-40$ amu) $\times 7$ polar angles & \\
$\left(22.5^{\circ}\right.$ & \\
\hline
\end{tabular}

The 'standard' operating mode will provide complete distribution function reports for five species, with oversampling near the spin axes removed by collapse, at intervals of 2-4 spins (12-24 s). In addition, crude moments and comprehensive mass spectra will be delivered at the intervals shown in the table. TIDE will remain in this mode during the bulk of its operations. Two exceptions are admitted:

High Time Resolution: When the selected criteria are met, which may include the presence of 'burst' data mode flags on the interfaces to EFI and HYDRA or the identification of high variability in the TIDE moment data, TIDE will switch automatically to a high time resolution mode, in which maximum array collapse is implemented and as much data as possible is sent every spin. Generally, this will include reduced resolution reports for each of a minimum of three species. Of course, TIDE can be left in this mode continuously, if that proves to be desireable. 
High Angular Resolution: When experience suggests that features are present in the data that are (a) unresolved in azimuth angle, (b) present at predictable locations and/or times, (c) persistent for multiple spins, and (d) have sufficient ion flux, TIDE can be programmed to sacrifice temporal resolution in favor of more detailed angular sampling. The angular sampling resolution can be increased from 32 bins $\left(11.25^{\circ}\right)$ to either 64 or 128 bins. This feature is expected to find its principle application in accurate measurement of cold polar wind temperature. Because there may be other unanticipated benefits as well, this mode will be exercised regularly, but on a low duty cycle.

\section{Summary}

The Thermal Ion Dynamics Experiment/Plasma Source Instrument (TIDE/PSI) has been developed in response to the requirements of the ISTP Program for a three dimensional plasma composition instrument capable of tracking the circulation of low-energy plasma throughout the polar magnetosphere, and investigating its participation in the formation of the diamagnetic hot plasma sheet and ring current plasma populations.

TIDE provides the sensitivity (seven channels at $\sim 1 \mathrm{~cm}^{2}$ each) and temporal resolution required for this purpose, as well as the capability of an electronicallycontrolled aperture to prevent overexposure in denser environments. Analogous to other optical systems, reduction of the TIDE aperture results in enhanced resolution of angular and energy features in the observed ion distribution, albeit at reduced counting rate and therefore reduced temporal resolution. TIDE automatically adjusts its sensitivity to varying conditions, and it emphasizes temporal or angular resolution when appropriate.

With nearly a full three-dimensional view of the sky each spin, TIDE's seven angular sectors/channels monitor all mass species simultaneously, eliminating the need for any sweeps except energy, and producing a 7-kByte image of the distribution function for each of five ion species once each 6-s spin. The TIDE DPU computes simple moments for each species every spin, and monitors the record for notable variability. It routinely encodes measurements in a floating point form, collapses angular oversampling out of the data array, and performs lossless compression to optimize telemetry use. When variability of the moments indicates the need for full time resolution, it truncates the data so as to obtain single spin temporal resolution for the most important ion species. Enhanced spin angular resolution may be obtained when fluxes are sufficient to permit 'stopping' down the aperture, at a corresponding loss of temporal resolution.

PSI produces a low-energy plasma local to the POLAR spacecraft which provides the ion current required to balance the photoelectron current, along with a low temperature electron population, thus regulating the spacecraft potential a few tenths of a volt positive. 
Thus TIDE/PSI will permit investigation of the extent to which ionospheric and low energy magnetosheath ions are lost to the solar wind or recirculated within the distant magnetotail neutral sheet. For those ions which are recirculated and energized, it will be possible to investigate (through collaboration with the TIMAS and other investigations) the mass-dependent degree of energization achieved as the plasma flows antisunward on merged flux tubes through the lobes and into the plasma sheet. TIDE/PSI will provide the fundamental information necessary to investigate the ionosphere and the magnetosheath as sources of low-energy plasma to the plasma sheet and ring current.

\section{Appendix 1. Mirror Shape}

Ideal optical mirrors are parabolic. This is the shape required to bring a parallel incoming photon beam through a focal line or point, in the cases of 1 or 2-D focusing, respectively. When this condition is met, the angular distribution of the external flux of photons is mapped by the mirror to a spatial image at the focal plane. The fact that the photons penetrate the mirror to a depth which is negligible in comparison to the system dimensions is essential to the simplicity of the parabolic focusing shape. Owing to the variation of charged particle penetration with angle of incidence onto an electrostatic mirror, it is clear that the form of an electrostatic mirror required for focusing departs from parabolic. However, the shape and placement of the mirror, with respect to the desired focal line or point, is not intuitively obvious.

Consider the geometry shown in Figure 17. The tangent to the front (grounded) grid, at an arbitrary point along it, is indicated along with a charged particle trajectory. The rear (biased) grid is assumed to be located at a fixed distance along the normal to the front (grounded) grid surface. The electric field between the grids points from the rear toward the front grid. Particles incident from the right, moving along the negative ' $y$ ' direction, feel no force until they pass through the front grid, apart from that due to grid fringe fields, which introduces some scattering. While between the two grids, these particles are under the influence of the applied electric field. Those particles with large enough velocity components along the field direction pass through the rear grid and are lost to the system. Those with smaller velocity along the field direction are turned by the field and directed toward the focal point, located at $(0, f / 2)$. We calculate the mirror geometry by determining the functional form of the front grid as $y(x)$ under the following simplifying assumptions:

(a) The grid is considered to be locally flat. That is, the slope of the curve $y(x)$ is considered to be constant over the region between a reflected ion's entry and exit points. This gives rise to a uniform electric field between the grids

$$
E=\frac{V_{m}}{D} \hat{n}
$$




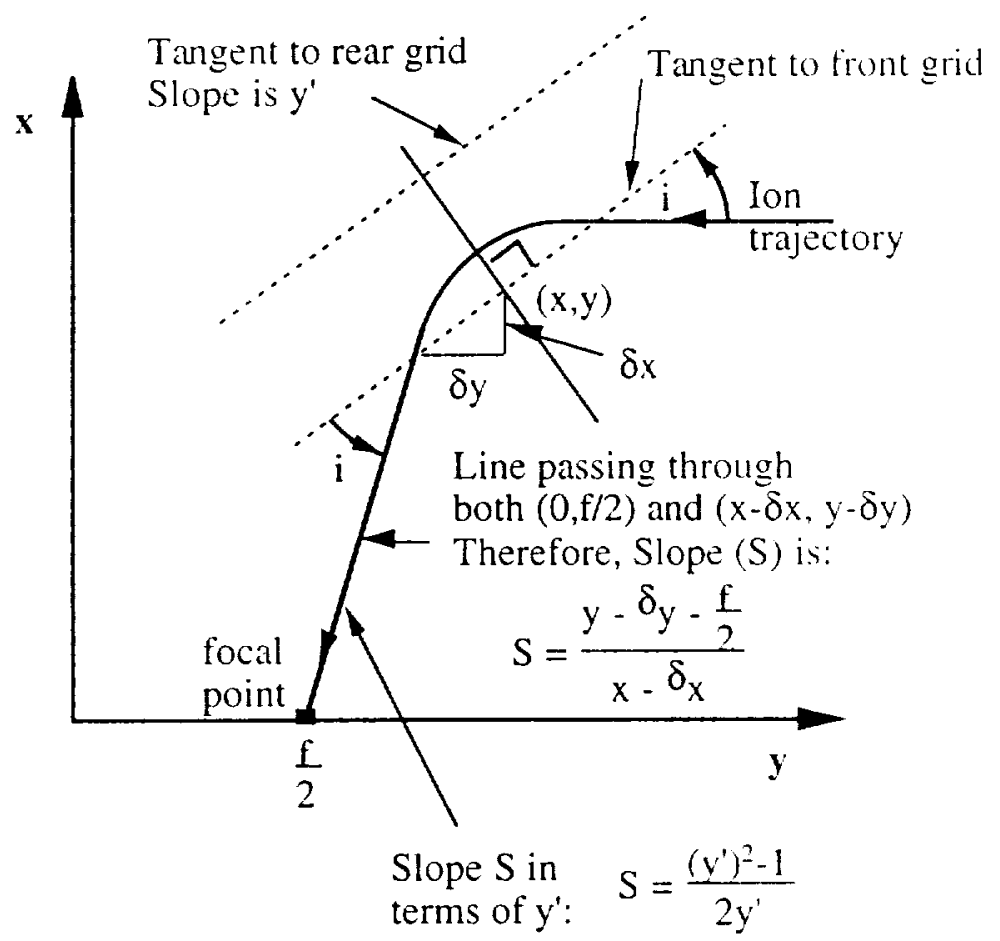

Fig. 17. Geometry for the derivation of the TIDE mirror shape required to bring a parallel beam, 6 incidentfrom the right, to a focus at the desired point $(0, f / 2)$.

where $V_{m}$ is the commandable applied mirror voltage, $D$ is the distance between the front and back grids, and $\mathrm{n}$ is the local normal to the front grid, pointing from back to front grids.

(b) The curve $y(x)$ is considered to be near enough to parabolic that a perturbation from the parabolic case provides a solution to the derived differential equation.

Assumption (1) above, in combination with the focusing condition and simple particle dynamics within the electric field region, leads to the following differential equation for the curve $y(x)$ :

$$
y=\frac{x}{2 y^{\prime}}\left(\left(y^{\prime}\right)^{2}-1\right)+\frac{R D}{\sqrt{\left(y^{\prime}\right)^{2}+1}}+\frac{f}{2},
$$

where $R$ is the ratio of the nominally selected ion kinetic energy per charge to applied mirror voltage $\left(E /\left(q V_{m}\right)\right)$, and $y^{\prime}$ is the slope of the curve $y(x)$ between the points of particle entry and exit. We have chosen a boundary condition such that, in the limit as $x$ approaches zero, the slope $y^{\prime}(x)$ should approach zero in the same manner as the parabolic solution does. That is, 


$$
\lim _{x \rightarrow 0}\left[y^{\prime}(x)\right]=\frac{x}{f} \quad \text { and } \quad \lim _{x \rightarrow 0}[y(x)]=R D \text {. }
$$

This nonlinear first order boundary value problem for $y(x)$ is solved through a perturbation technique, using the second approximation above, as described by Pollock et al. (1994).

In the TIDE instrument, the following parameters were selected for mirror construction:

$$
f=56.7 \mathrm{~mm}, \quad D=5 \mathrm{~mm}, \quad R=1.60 .
$$

The basis for these choices is as follows: In order that the intergrid spacing be large compared with the grid wire spacing of $0.036 \mathrm{~mm}$ (necessary for small fringe field scattering effects), $D$ was chosen to be 14 times as large. In order that the focusing direction angular response of TIDE be a nominal $10^{\circ}$, and in view of the $10 \mathrm{~mm}$ aperture of the TOF section, the focal length was chosen as $10 \mathrm{~mm} \times$ $\tan \left(10^{\circ}\right)=56.7 \mathrm{~mm} . R$ is chosen to provide optimal focusing at an energy 'typical' of TIDE operation, which may extend from approximately one to two times the mirror potential.

\section{Appendix 2. Electronics Description}

Figure 5 is a simplified schematic diagram of the major electronic components of TIDE. The actual TIDE operational power is $9.1 \mathrm{~W}$, well under the allocation of $10.0 \mathrm{~W}$. The major TIDE electronics subsystems are: spacecraft power and data interfaces; low voltage power supplies and filters $( \pm 3, \pm 5, \pm 10, \pm 15$, and $28 \mathrm{~V})$; high voltage power supplies ( 0 to $\pm 300 \mathrm{~V} \mathrm{RPA}, 0$ to $\pm 300 \mathrm{~V}$ Mirror, 0 to $-2.4 \mathrm{kV}, 0$ to $-3.6 \mathrm{kV}, 0$ to $-15 \mathrm{kV})$, TOF electronics, TOF-ADC interface, DPU and associated memory, and Ground Support Equipment (GSE). The TIDE electronics are built in a modular fashion such that, to the greatest extent possible, similar groups of electrical functions are on the same electronics board, as briefly summarized in Table VI.

\section{TIME-OF-FLIGHT ELECTRONICS}

Seven START and seven STOP signal lines provide inputs to the TOF electronics. The seven STOP signals are summed into a single STOP signal, effectively a logical OR operation. Amplitude discrimination of MCP pulses occurs on each START and the summed STOP signals, resulting in eight pulse trains which are accumulated regardless of time correlation to form START and STOP 'singles' rates. The seven STARTS are summed (OR'd) after discrimination.

Correlated START/STOP pulse pairs trigger the capture of output from a single time-to-amplitude converter (TAC) circuit, which, owing to the logical OR operations, can service the seven TIDE polar-angle sectors. Figure 18 shows a simplified 
TABLE VI

Electronics board functions

\begin{tabular}{cl}
\multicolumn{2}{c}{ Electronics board functions } \\
\hline Board & Function \\
\hline 1 & $-3.6 \mathrm{kV},+0.30 \mathrm{kV}$ HVPS \\
3 & $-2.4 \mathrm{kV},+1.30 \mathrm{kV}$ HVPS \\
- & $-15.0 \mathrm{kV}$ HVPS \\
4 & (shield) \\
5 & Filters and housekeeping LVPS \\
- & TOF and DPU LVPS \\
6 & Phield) \\
7 & Power supply D/A and HV enable \\
8 & Spacecraft I/F \\
9 & Processor (IMP and DP) \\
10 & Processor RAM (144 kB) \\
11 & Processor ROM (72 kB) \\
12 & Bulk RAM (384 kB) \\
13 & TOF memory matrix (64 kB) \\
14 & TOD direct event memory matrix (8 kB) \\
15 & TOF load control \\
16 & TOF accumulator and pulser \\
17 & TOF electronics (with 15 daughter cards) \\
\hline
\end{tabular}

schematic diagram of the TIDE TOF logic. The mass identification process consists of converting a time interval between received START and STOP pulses into a digital word and combining this information with the encoded polar angle of arrival (START sector ID). Such events are accumulated in memory addresses corresponding to the TOF, the START sector ID, the current energy step, and current spin sector. Additional information is obtained from the singles rates to monitor total ion flux (independent of mass/q) and TOF processing efficiency. Error and reset logic are used to prevent data collisions and corruption during the processing interval.

\section{TOF TEST PULSER}

A pulser circuit is included in the TOF electronics section to directly stimulate the START and STOP preamplifiers in all seven sectors. Pulses from the preamplifiers are used to test the entire signal chain. The pulser generates a range of selected delays between START and STOP pulses in order to test TOF circuitry. These TOF data are fed into the DPU memory and are used to test DPU routines. 

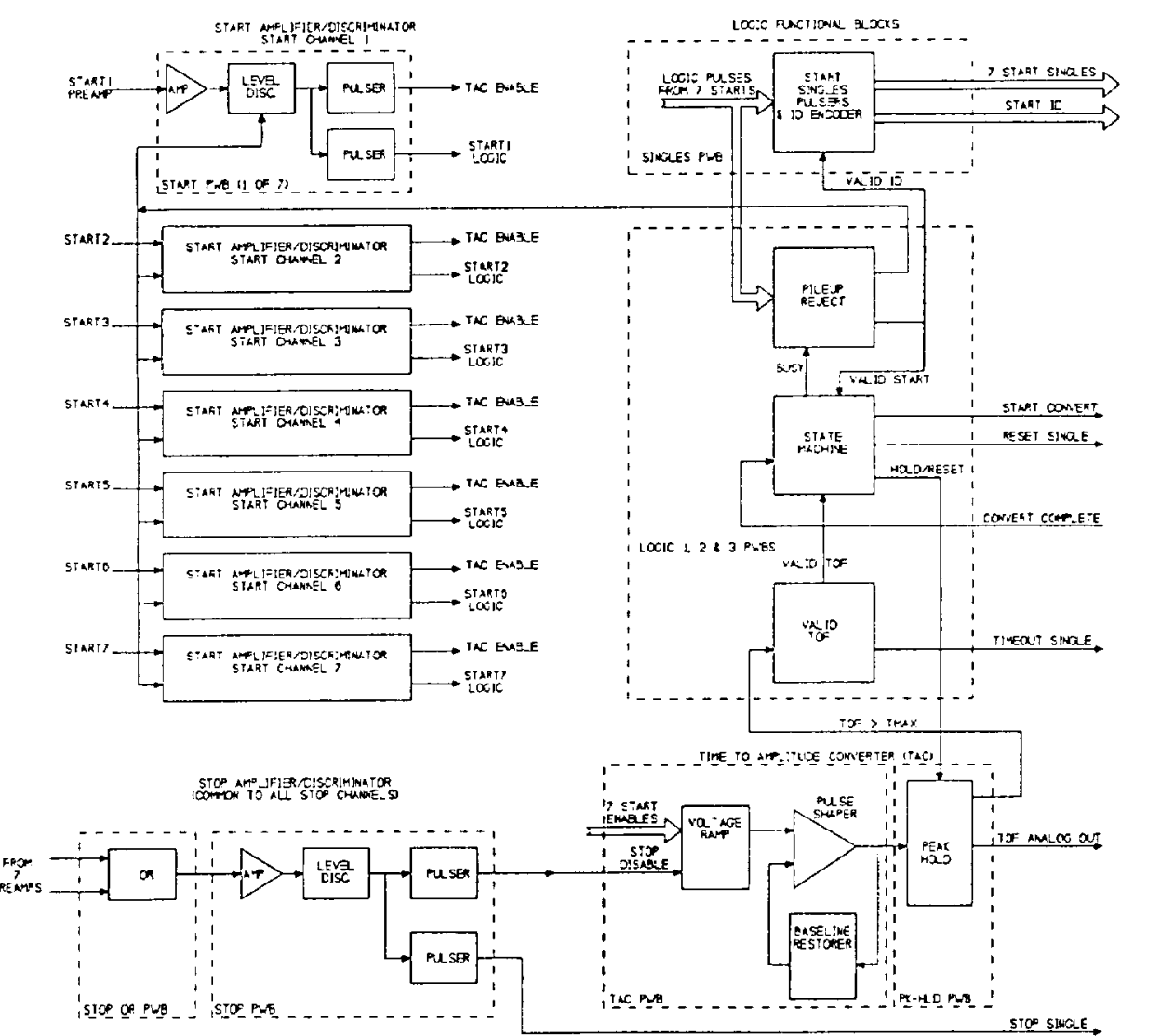

Fig. 18. TOF electronic block diagrams illustrating the relationships among the principal components: the START and STOP preamplifier-discriminators, the TAC, and the logic module.

\section{ADC FOR TAC SIGNAL}

The ADC output determines the bin location for sorting TOF according to time channels prior to count rate transmission. For TIDE this is 8 bits or 256 channels. The number of mass peaks selected from the TOF channels is limited by telemetry to five $M / Q$ peaks.

\section{HIGH VOLTAGE POWER SUPPLIES}

Table VII summarizes the TIDE high voltage power supplies and the requirements for these supplies, which are further discussed in the subsections below.

\section{TIME-OF-FLIGHT HVPS}

Power to the STOP MCPs mounted at the TOF HV is provided by a direct tap on the $-15.0 \mathrm{kV}$ supply. Although this requires an extra HV wire and feedthroughs 
TABLE VII

High voltage power supply specifications

\begin{tabular}{|c|c|c|c|c|c|c|}
\hline Label & Range & Resol. & Ripple & Drift & Monitor & Comments \\
\hline TOF & 0 to $-15.0 \mathrm{kV}$ & 8 bits & $\pm 1 \%$ & $\pm 1 \%$ & $\begin{array}{l}0-4.00 \mathrm{~V}, \\
8 \text { bits }\end{array}$ & $\begin{array}{l}\text { 2nd tap @ } 14.2 \mathrm{kV} \text { nominal } \\
\text { armed, enabled AND comanded }\end{array}$ \\
\hline $\begin{array}{l}\text { START } \\
\text { MCP }\end{array}$ & 0 to $-3.6 \mathrm{kV}$ & 8 bits & $\pm 0.5 \%$ & $\pm 2 \%$ & $\begin{array}{l}0-4.0 \mathrm{~V} \\
8 \text { bits }\end{array}$ & $\begin{array}{l}\text { Load: } 85 \mu \mathrm{A} \\
\text { armed, enabled, AND commanc }\end{array}$ \\
\hline $\begin{array}{l}\text { STOP } \\
\text { MCP }\end{array}$ & 0 to $-2.4 \mathrm{kV}$ & 8 bits & $\pm 0.5 \%$ & $\pm 2 \%$ & $\begin{array}{l}0-4.0 \mathrm{~V} \\
\text { ! bits }\end{array}$ & $\begin{array}{l}\text { Load: } 85 \mu \mathrm{A} \\
\text { armed, enabled, AND commanc }\end{array}$ \\
\hline RPA & 0 to $300 \mathrm{~V}$ & 12 bits & $\begin{array}{l} \pm 1 \% \text { or } \\
\pm 50 \mathrm{mV} \\
\omega<5 \mathrm{~V}\end{array}$ & $\pm 1 \%$ & $\begin{array}{l}0-4.0 \mathrm{~V}, \\
8 \text { bits }\end{array}$ & $\begin{array}{l}\text { Settle to } 5 \% \text { in } 0.6 \mathrm{~ms}, \Delta V<6 \\
\text { No resistive load }\end{array}$ \\
\hline Mirror & 0 to $300 \mathrm{~V}$ & 8 bits & $\begin{array}{l} \pm 1 \% \text { or } \\
\pm 50 \mathrm{mV} \\
\Theta<5 \mathrm{~V}\end{array}$ & $\pm 1 \%$ & $\begin{array}{l}0-4.0 \mathrm{~V} \\
8 \text { bits }\end{array}$ & $\begin{array}{l}\text { Settle to } 5 \% \text { in } 0.6 \mathrm{~ms}, \Delta V<6 \\
\text { Controlled as } 8 \text {-bit fraction of th } \\
\text { current mirror supply potential } \\
\text { No resistive load }\end{array}$ \\
\hline
\end{tabular}

to the sensor, it lowers total power requirements by eliminating the low ohmic divider. The remainder of the HV values needed for the TOF are provided by a high ohmic $\left(>10^{10} \Omega\right.$ ) divider mounted inside the sensor housing. The supply should be commanded to high voltage in a number of steps in order to allow the sensor to condition itself to the applied voltage, and also to provide a lower voltage to operate at in case we cannot achieve the $-15 \mathrm{kV}$ maximum. As is the case for other HV supplies on TIDE there are 8 bits of usable control available in the DAC and buffer registers so that a resolution of $58.6 \mathrm{~V}$ can be used to adjust the TOF high voltage. The $-15.0 \mathrm{kV}$ supply monitor provides an analog voltage scaled at 0 to $4 \mathrm{~V}$ for 0 to $-15.0 \mathrm{kV}$ ( $5000: 1$ divider). The monitor voltage is digitized to 8 bits of accuracy. The time sequence for raising the TOF HV to $-15.0 \mathrm{kV}$ consists of two stages. First, the instrument must be exposed to high vacuum for a duration sufficient to insure thorough outgassing of the entire instrument. In practice, we have had good success by turning on TOF high voltage only after the instrument has been at a chamber pressure of $\leq 1 \times 10^{-6} \mathrm{~T}$ for a minimum of 24 hours.

\section{MICROCHANNEL PLATE HVPS}

The START and STOP MCPs are operated from separate HVPS for reliability and because each stack must run at a different potential in any case. In order to avoid individual high voltage bias supplies for each sector while maintaining good uniformity from sector to sector, the MCPs have been specified to have consistent impedances from plate to plate and stack to stack $( \pm 10 \%)$. The resistance per 
plate is approximately: $R \sim 100 \mathrm{M} \Omega$. Thus a single START stack ( 3 plates) is $\sim 300 \mathrm{M} \Omega$, and the STOP stack ( 2 plates) is $\sim 200 \mathrm{M} \Omega$. Since a stack of 3 START MCP plates has a resistance of $\sim 300 \mathrm{M} \Omega$, a full set of 7 in parallel has a total resistance of $R \sim 43 \mathrm{M} \Omega$ and at $-3.6 \mathrm{kV}$ maximum voltage the total strip current is $\sim 85 \mu \mathrm{A}$. The stack of 2 STOP MCPs has a resistance of $200 \mathrm{M} \Omega$, so that a full set of 7 in parallel gives $R \sim 29 \mathrm{M} \Omega$. At $-2.4 \mathrm{kV}$ the total strip current is $\sim 85 \mu \mathrm{A}$.

MCP Z stacks are chosen for consistent multiplication gains, by dividing into START, STOP, and SPARE groups. During calibration, the optimum MCP bias operating points for START and STOP detectors is determined by varying the biases and observing the integral rate of discriminated pulses.

\section{RETARDING POTENTIAL ANALYZER SUPPLY}

The voltage varies from $0.00 \mathrm{~V}$ to $300.0 \mathrm{~V}$ with 12-bit resolution. This corresponds to a voltage resolution $\Delta V=0.0732 \mathrm{~V}$. The shortest sample period is $5.86 \mathrm{~ms}$ and settling time is $<10 \%$ of this period. The RPA supply output monitor provides an analog signal scaled at 0 to $4.0 \mathrm{~V}$ for 0 to $300 \mathrm{~V}$ input. This is monitored in TM to 8 bits of accuracy.

\section{MIRROR SUPPLY}

The voltage varies from $0.0 \mathrm{~V}$ to $300 \mathrm{~V}$, but is controlled as a fraction of the RPA potential ranging from zero to unity with 8 bits of resolution. Thus the default is for the Mirror to track the RPA potential in a fixed ratio during a sweep. However the ratio is commandable on each step of the energy sweep, and is the parameter which controls the overall instrument sensitivity. The mirror voltage is the specified fraction of the RPA voltage to an accuracy equal to that of the 8-bit command resolution $(0.4 \%)$ or $40 \mathrm{mV}$, whichever is greater.

\section{DATA PROCESSING UNIT}

The following describes the general functions of the DPU. Figure 19 shows a highly schematic overview of the TIDE DPU and control system.

\section{Microprocessors}

The data processor (DP) is a Sandia SA3300 microprocessor with a 16-bit external and 32-bit internal bus, running at $8 \mathrm{MHz}$. This processor is used to execute computation intensive tasks, primarily the calculation of moments from the spin by spin data arrays. Hardware capability is provided to interrupt the processor on critical Levents such as the spin pulse, minor frame, etc.

The instrument mode processor (IMP) is also a Sandia SA3300 microprocessor running at $5 \mathrm{MHz}$. It is used to perform housekeeping tasks such as telemetry formatting, HV control and associated TIDE control functions. In addition, the 


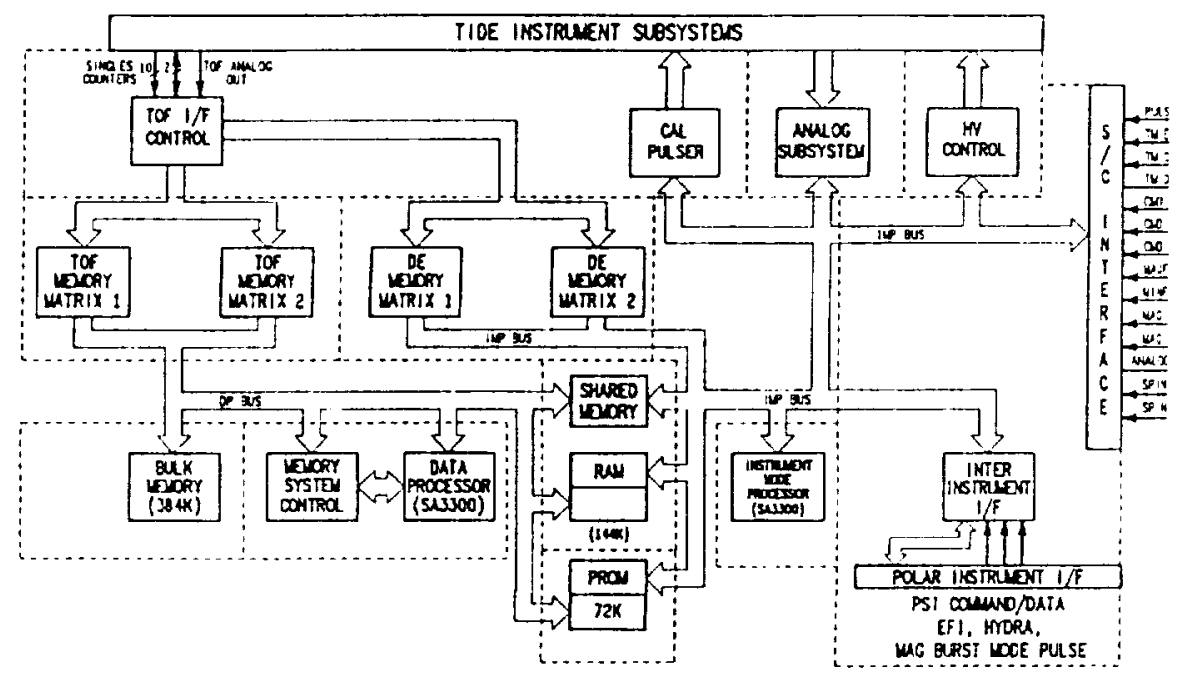

Fig. 19. TIDE IMPIDP schematic block diagram.

IMP performs bulk data compression when that function is enabled. Interrupt capability is also be provided for events such as minor frame sync, command, telemetry, etc.

Memory configuration is as summarized in Table Vl. The IMP and the DP have shared access to $144 \mathrm{kB}$ of RAM and $72 \mathrm{~KB}$ of PROM. The processors obtain their data through data acquisition memories which are incremented independently of the processors by the load control circuit. These amount to $8 \mathrm{kB}$ and $64 \mathrm{kB}$ for the JMP and DP, respectively. In addition, the DP has a bulk RAM allocation of 384 $\mathrm{kB}$ for intensive calculation space.

\section{TOF INTERFACE}

The TOF interface requires digitizing the TOF signal by a flash ADC to 8-bits resolution. The 8 bits are then filtered through a mass/charge lookup table, which is RAM based and can be updated in flight, that generates a 3-bit mass address. Along with a 3-bit sector identification, these data are binned according to azimuth and energy. These data are acquired in a 128 kilobyte memory system over a 6-5 spin period. There are two such memory systems so that at a spin boundary the memory systems are swapped. This allows the data processor access to the previous spin of data while acquisition is occurring in the other memory system.

The ADC digitizes the analog TOF signal to an 8-bit channel number, which determines the bin location for sorting events, both in Direct Event memory (full 8-bit resolution) or in the main Angle-Energy array memory (3-bit resolution). The ADC input must have an input impedance sufficiently high that it is adequately driven by the TOF output driver. 
16-bit counters are provided to accumulate all singles rate outputs from the TOF electronics, sorted according to azimuth angle and energy step. These data are acquired in the same memory system as described above. The 8-bit output of the TOF ADC along with the 3-bit sector ID is used in a separate (direct event) data acquisition system that bins the 11-bit data over a 3-min accumulation time. Upon an ADC start pulse, each direct event is binned during a dead time period which exceeds that for the binning of ordinary TOF events into mass bins, as indicated in Table III. To accommodate the longer accumulations, 32 bit accumulation words are used.

\section{MEMORY SUB-SYSTEM}

Hardware capabilities are provided to the data processor for copying memory contents from the data acquisition memory to general purpose memory. In addition to the copy function, a hardware lookup table function is supplied to encode the data in a compact floating point representation. Finally, a hardware block add/subtract function is provided. All of these hardware functions are under software control and are intended to reduce software overhead of the data processor.

\section{HIGH VOLTAGE POWER SUPPLY INTERFACE}

Logic is provided to turn on the HV power supplies in three stages. Spacecraft pulse commands are used to arm the supplies for microprocessor commanding. The three pulse commands arm the RPA/MIR, MCP (START and STOP) and TOF high voltage supplies. A fourth pulse command is provided to act as a 'breaker' switch for all HV supplies. Once the supplies have been armed, (major) commands can then be issued to individually enable commanding any of the five HV supplies. Finally, the appropriate control levels, which are generated by digital-to-analog converters (DACs), can be commanded (minor commands) to complete the HV turn-on. Upon DPU power up the control levels reset to zero. Control of the RPA and MIRROR HV control levels is done via sweep tables which are either resident in ROM or built up in RAM by (minor command) upload and then executed by minor command. The stepping of the supplies according to the tables is synchronized to the sample period. The RPA and MIRROR HV have 12-bit and 8-bit control voltage resolution, respectively. The Start MCP, Stop MCP, and TOF HV have 8 -bit control voltage resolution.

\section{SPACECRAFT INTERFACE}

All of the spacecraft interfaces described are in accordance the GE General Instrument Interface Specification (GIIS) document number 3282065. The interface consists of a 40 -bit serial command channel, together with three 8-bit parallel/serial registers that are provided for the $\mathrm{s} / \mathrm{c}$ to acquire telemetry data on greater than 1 millisecond centers per minor frame. MAGAZ and MAGEL signals are received 
from the s/c. The spin phase clock and pulse are used to synchronize the operations of the instrument to the s/c spin. Analog housekeeping signals for ascertaining the health and safety of the instrument are digitized using a general purpose analog to digital converter. A PSI 'Squirt' signal is provided to the Electric Field and Plasma Wave Instruments. Signal interfaces are provided to the HYDRA (energetic plas$\mathrm{ma}$ ) and EFI (Electric Field Instrument) investigations in order to determine when

they enter a 'burst memory' state indicative of rapid temporal fluctuations in the local plasma or fields.

GSE

The GSE is based on a functional emulation approach. It contains separate PC computers to simulate the POLAR spacecraft and the controlling ground station. It also contains a Sun workstation to serve as an emulator for the Remote Data Analysis Facility, where the full data displays are developed. This is a complex system, but the approach is designed to test all phases of the data link between TIDE and the investigators. All power supply, pulser calibration, and similar functions are computer controlled by the GSE computer. There is never a need for persons conducting a test to 'turn a knob' - the setting of which then has to be written down or otherwise entered manually into the test data record.

\section{PSE ELECTRONICS}

The PSI electronics system is illustrated schematically in Figure 20. It contains the discharge, keeper, heater, and bias supplies for operation of the source, a bipolar log-electrometer to measure the net emission current from the source (this constitutes the return current from the satellite), valve drivers, analog telemetry signal conditioning, and the TIDE interface.

The remaining components of the PSI are the various control electronics and interface circuitry necessary for interfacing with the TIDE power, data, and command system. The primary controls are the startup heater, the keeper potential, anode potential, and the bias supply. The primary data reported to the data stream are the emission current and power supply monitors, which are reported in the TIDE data stream.

The TIDE instrument controls all phases of PSI operations. The application of $28-\mathrm{V}$ power and a simple command is all that is required to start the plasma source. A maximum of 20 other commands are required to set the level of the bias supply and make minor adjustments to the operation of the source (including control of all power supplies). Approximately 20 telemetry words are used to monitor PSI operations. 


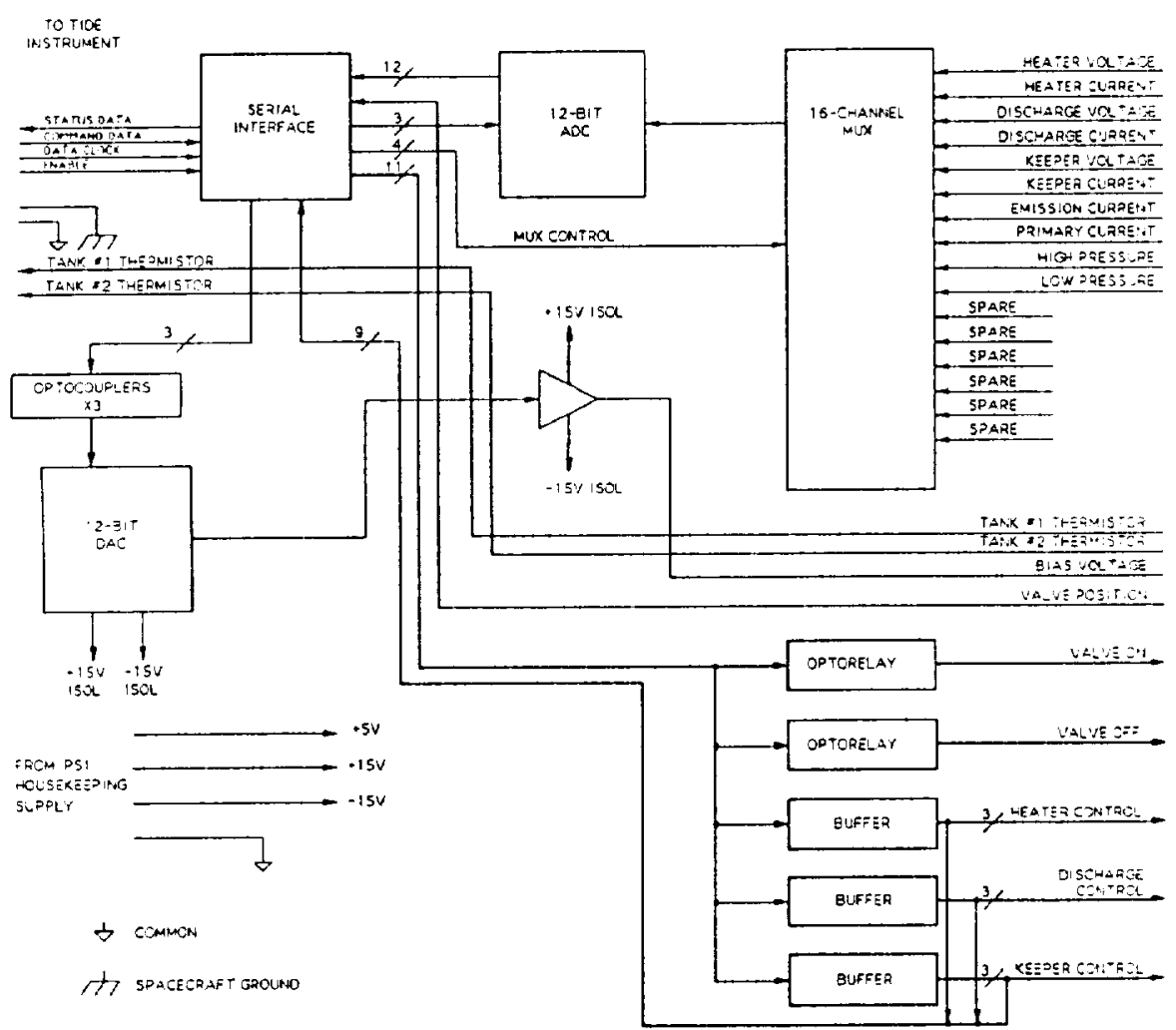

Fig. 20. PSI electronics block diagram.

\section{Acknowledgements}

The authors wish to acknowledge the crucial efforts of John Galloway, GGS instrument manager for TIDE/PSI; Bill Chisholm, Vic Coffey, and Nelson Martinez of MSFC; Peggy Sloan and Dick West of Boeing Computer Support Services; Ron Bowman, Ron Black, Al Blevins, Poul Jensen, Dana Kloza, Don Shirley, Kirk Smith, and David Strain of Southwest Research Institute; Kevin McCabe of Los Alamos National Laboratory; Todd Williarnson of Hughes Research Laboratories; as well as other members of the technical and science staffs of the respective organizations. All labored long and hard at one time or another to make TIDE what it is. We are indebted to M. Torr, M. Neugebauer, and W. Lennartsson for stimulating discussions concerning the design of electrostatic mirrors; and to R. C. Olsen for advice and discussion regarding the plan for the PSI. A. P. Biddle, J. H. Hoffman, R. A. Heelis, and W. W. Wright contributed to early versions of the Mirror/RPA design. SwRI work was carried out under NASA grants NAG8-114 and NAG8693, NASA contract NAS8-38189, and SwRI Internal Research Project 15-9455. Work performed at Los Alamos National Laboratories was carried out under the 
auspices of the U.S. Dept. of Energy. Work performed at CRPE was funded by CNES.

\section{References}

Chandler, M. O., Waite, J. H., Jr., and Moore, T. E.: 1990, J. Geophys. Res. 96, 1421.

Chappell, C. R.: 1988, Rev. Geophys. Space Phys. 26, 229.

Chappell, C. R., Fields, S. A., Baugher, C. R., J. H., Hoffman, J. H., Hanson, W. B., Wright, W. W., Hammack, H. D., Carignan, G. R., and Nagy, A. F.: 1981, Space Sci. Instrum. 5, 477.

Delcourt, D. C., Sauvaud, J. A., and Moore, T. E.: 1990, J. Geophys. Res. 95, 20937.

Giles, B. L., Chappell, C. R., Moore, T. E., Comfort, R. H., and Waite, J. H., Jr.: 1994, 'Statistical Survey of Pitch Angle Distributions in Core (0-50 eV) Ions from Dynamics Explorer 1: Outflow in the Auroral Zone, Polar Cap, and Cusp', J. Geophys. Res. 99(A9), 17483.

Lockwood, M., Chandler, M. O., Horwitz, J. L., Waite, J. H., Jr., Moore, T. E., and Chappell, C. R.: 1985, J. Geophys. Res. 90, 9736.

Moore, T. E.: 1991, Rev. Geophys. Suppl., 1039.

Moore, T. E. and Young, D. T.: 1993, TIDE/PSI Flight Hardware Specifications, MSFC Document TIDE-FHWS-04.

Nagai, T., Johnson, J. F. E., and Chappell, C. R.: 1983, J. Geophys. Res. 88, 6944.

Olsen, R. C.: 1981, J. Spacecraft and Rockets 18, 462.

Olsen, R. C.: 1985, J. Spacecraft and Rockets 22, 254.

Pollock, C. J., Chandler, M. O., Moore, T. E., Waite, J. H., Jr., Chappell, C. R., and Gurnett, D. A.: 1990, J. Geophys. Res. 95, 19969.

Pollock, C. J., Moorre, T. E., and Reasoner, D. L.: 1994, 'Quasi-parabolic Electrostatic Charged Particle Mirror', Rev. Sci. Instr. (submitted).

Young, D. T.: 1989, in J. H. Waite, Jr., J. L. Burch, and R. L. Moore (eds.), 'Space Plasma Mass Spectrometry Below $60 \mathrm{keV}$ ', Solar System Plasma Physics, Geophysical Monograph No. 54, Am. Geophys. Un., Washington, D.C., p. 143. 